\title{
Les traces d'une occupation du Néolithique moyen, de tradition Castellic, dans le bassin de Rennes (Bretagne, France)
}

Traces of a Middle Neolithic occupation of the Castellic tradition in the area of Rennes

Spuren einer mittelneolithischen Besiedlung in der Tradition der CastellicGruppe im Becken von Rennes (Bretagne, Frankreich)

Las huellas de una ocupación del Neolítico medio de tradición Castellic en la cuenca de Rennes (Bretaña, Francia)

Vérane Brisotto, Xavier Henaff et Stéphane Bourne

\section{(2) OpenEdition}

Édition électronique

URL : https://journals.openedition.org/rao/2900

DOI : $10.4000 /$ rao. 2900

ISBN : 978-2-7535-5014-8

ISSN : $1775-3732$

Éditeur

Presses universitaires de Rennes

Édition imprimée

Date de publication : 31 décembre 2015

Pagination : 59-81

ISBN : 978-2-7535-5012-4

ISSN : 0767-709X

Référence électronique

Vérane Brisotto, Xavier Henaff et Stéphane Bourne, «Les traces d'une occupation du Néolithique moyen, de tradition Castellic, dans le bassin de Rennes (Bretagne, France) », Revue archéologique de I'Ouest [En ligne], 32 | 2015, mis en ligne le 28 avril 2018, consulté le 22 août 2022. URL : http:// journals.openedition.org/rao/2900 ; DOI : https://doi.org/10.4000/rao.2900 


\title{
Les traces d'une occupation du Néolithique moyen, de tradition Castellic, dans le bassin de Rennes (Bretagne, France)
}

\author{
Traces of a Middle Neolithic Occupation of the Castellic Tradition \\ in the Area of Rennes (Brittany, France)
}

\author{
Vérane Brisotto ${ }^{a}$, Xavier Henaff $^{b}$ et Stéphane Bourne ${ }^{a}$
}

\begin{abstract}
Résumé : Suite à la création de la ZAC des Champs-Bleus, sur la commune de Vezin-le-Coquet (Ille-et-Vilaine), les différents diagnostics archéologiques réalisés entre 2006 et 2011 ont permis de mettre en évidence une occupation néolithique qui, bien que difficilement caractérisable, a livré du mobilier céramique de tradition Castellic, ainsi qu'un petit assemblage lithique et macro lithique pouvant se rattacher au Néolithique moyen II régional. Cette série constitue une première dans le bassin de Rennes et permet de poser un jalon géographique pour cette période, à mi-chemin entre les sites de la zone nucléaire localisée autour du golfe du Morbihan, ceux de la côte nord, et les quelques indices attestant de son rayonnement en Normandie et dans les îles Anglo-Normandes.
\end{abstract}

\begin{abstract}
Following the creation of the ZAC des Champs-Bleus in the municipality of Vezin-le-Coquet (Ille-et-Vilaine, Bretagne, France), different archaeological evaluations were carried out between 2006 and 2011. They have brought to light a Neolithic occupation which, although not easily characterized, includes pottery and stoneworking typical of the Castellic, a Middle Neolithic II regional group. This small series represents a first in the Rennes area, adding another site to the sites already discovered around the Gulf of Morbihan and on the north coast as well as in Normandy and the Channel Islands.
\end{abstract}

Mots clés : Néolithique moyen, Bretagne, Castellic, céramique, outillage lithique et macro-lithique, bâtiment.

Keywords: Middle Neolithic, Brittany, Castellic, pottery, stoneworking and macrolithique tools, building.

\section{Circonstances et historique DES OPÉRATIONS}

Dans le cadre de la création d'une ZAC à Vezin-le-Coquet, à l'ouest de Rennes, le Service régional de l'Archéologie de Bretagne a prescrit trois diagnostics, qui ont été réalisés par l'Inrap en 2006, 2008 et 2011 (fig. 1).

La première opération, menée en 2006, s'est révélée assez pauvre archéologiquement. Elle a toutefois mis en évidence quelques indices néolithiques, avec la découverte hors structure d'un tesson décoré associé à un grattoir sur éclat laminaire de silex beige. Trois autres tessons, dont une anse provenant d'une dépression sédimentaire, peuvent également se rattacher à cette période (Tournier, 2006).

La seconde opération, effectuée en 2008, a été réalisée sur une surface de 156266 mètres carrés. Outre quelques indices de l'âge du Bronze et de l'âge du Fer, elle a révélé la présence d'une occupation néolithique, qui sera l'objet principal de cette présentation (Brisotto, 2008). La découverte d'une grande fosse, renfermant plusieurs formes céramiques, très fragmentées mais

a Inrap Grand-Ouest, 37 rue du Bignon, CS 67737, 35577 CESSON-SÉVIGNÉ.

b Inrap Grand-Ouest, Centre Archéologique de Carquefou, 4 rue du Tertre, 44477 CARQUEFOU. 


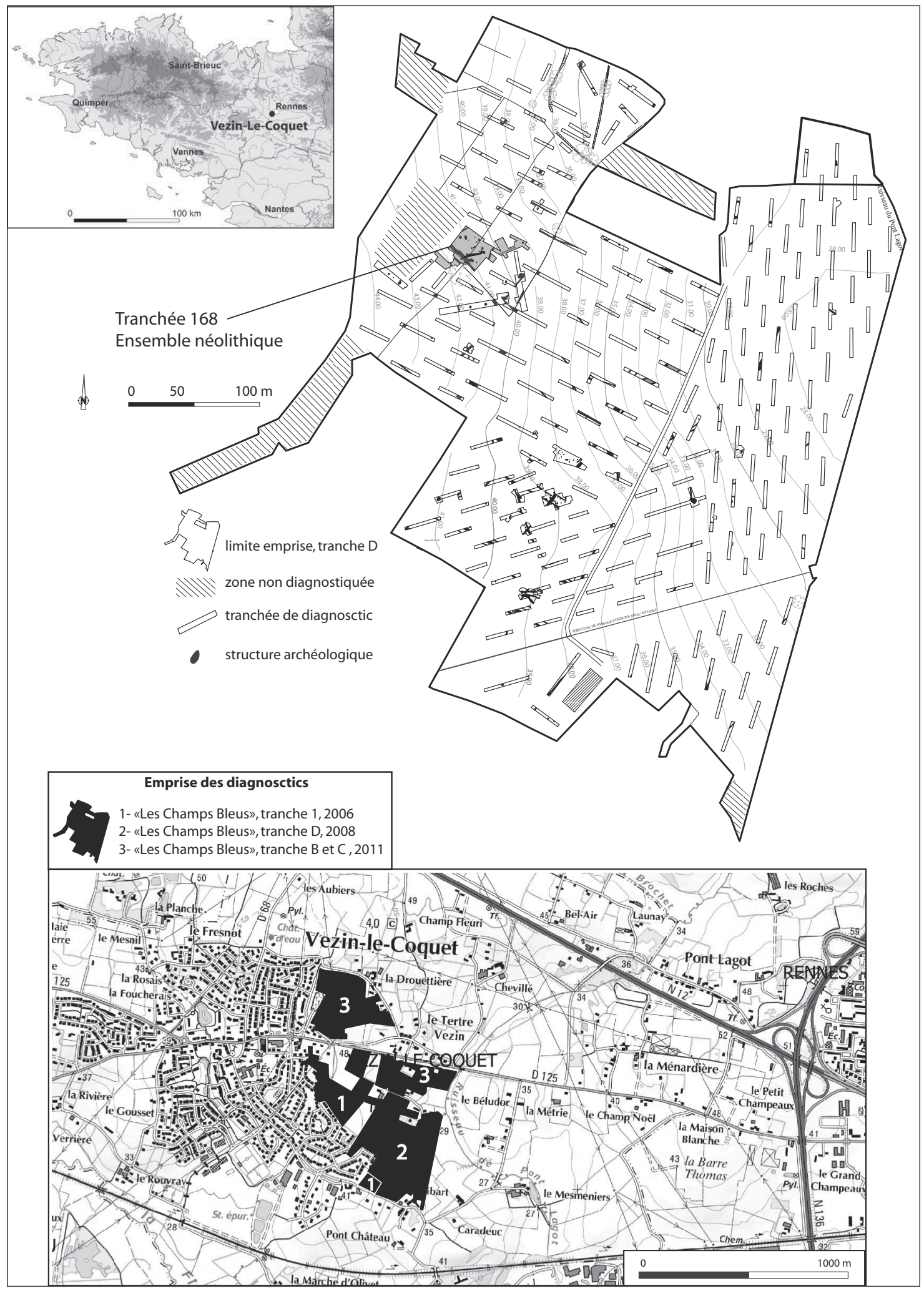

Figure 1 : Vezin-le-Coquet "Les Champs Bleus " : localisation géographique des diagnostics sur fond de carte IGN, et plan général de la tranche D, 2008. DAO A. Desfonds, V. Brisotto.

Figure 1: Vezin-Le-Coquet, "Les Champs Bleus", geographic location of the evaluation on the IGN map and general plan of sector D, 2008. 
dont certaines sont décorées, a motivé une extension du décapage. Dans cette zone de près de mille mètres carrés, quelques fosses et/ou trous de poteau ainsi qu'une structure allongée ont été mis au jour. Au regard des vestiges découverts, difficilement perceptibles et de leur proximité avec la limite d'emprise, il a été convenu, en accord avec le SRA et L. Laporte, membre de la CIRA Bretagne, de fouiller intégralement ce petit ensemble dans le cadre du diagnostic.

La fouille de ces structures a livré un assemblage céramique, lithique et macro-lithique homogène, datable du Néolithique moyen II. Quatre prélèvements de charbons de bois ont été effectués en vue de faire réaliser des datations par le radiocarbone.

Enfin, une petite série lithique pouvant se rattacher à la même occupation a été collectée hors contexte lors d'un troisième diagnostic réalisé en 2011. Sur cette même emprise, des traces ténues d'occupation à l'âge du Bronze, l'âge du Fer, et au Moyen Âge ainsi que deux structures à pierres chauffées non datées mais évoquant les dispositifs désormais bien reconnus pour le Néolithique (Brisotto, 2011).

Enfin, un toponyme de lieu-dit proche de l'emprise, Le Tertre, évoque lui aussi les périodes anciennes et leurs architectures funéraires.

\section{Présentation des Structures néolithiQues (FIG. 2)}

Les vestiges néolithiques mis au jour lors du diagnostic de 2008 occupent le replat d'un versant relativement marqué, orienté nord-est/sud-ouest. Ils surplombent à environ $40 \mathrm{~m}$ NGF le fond d'un vallon, où coule le ruisseau du Pont Lagot, à environ trois cents mètres plus au nord.

Géologiquement, l'emprise du diagnostic se situe en bordure d'un placage de limon fin homogène recouvrant le socle briovérien constitué d'une alternance siltowakckeuses dures vertes ou grises. Sur le terrain, les vestiges néolithiques se situent à 0,60 mètre de profondeur. Ils sont scellés par deux niveaux de limon argileux gris à gris-brun-orangé, résultant probablement d'un phénomène de colluvionnement. Le fond des structures atteint des niveaux d'altérites ou ponctuellement de siltites vertes grisâtres plus ou moins altérées.

La première structure mise au jour est une grande fosse de forme ovale (fig. 2 et 3). Dès le décapage, la présence en surface de cette fosse, de tessons de facture néolithique a suscité l'extension de la tranchée. D'autres anomalies sédimentaires sont alors apparues. Leurs contours difficilement perceptibles n'ont été décelés qu'après des nettoyages successifs et à la faveur d'une lumière favorable. Leurs comblements se distinguaient nettement de ceux des bioturbations et des structures modernes.

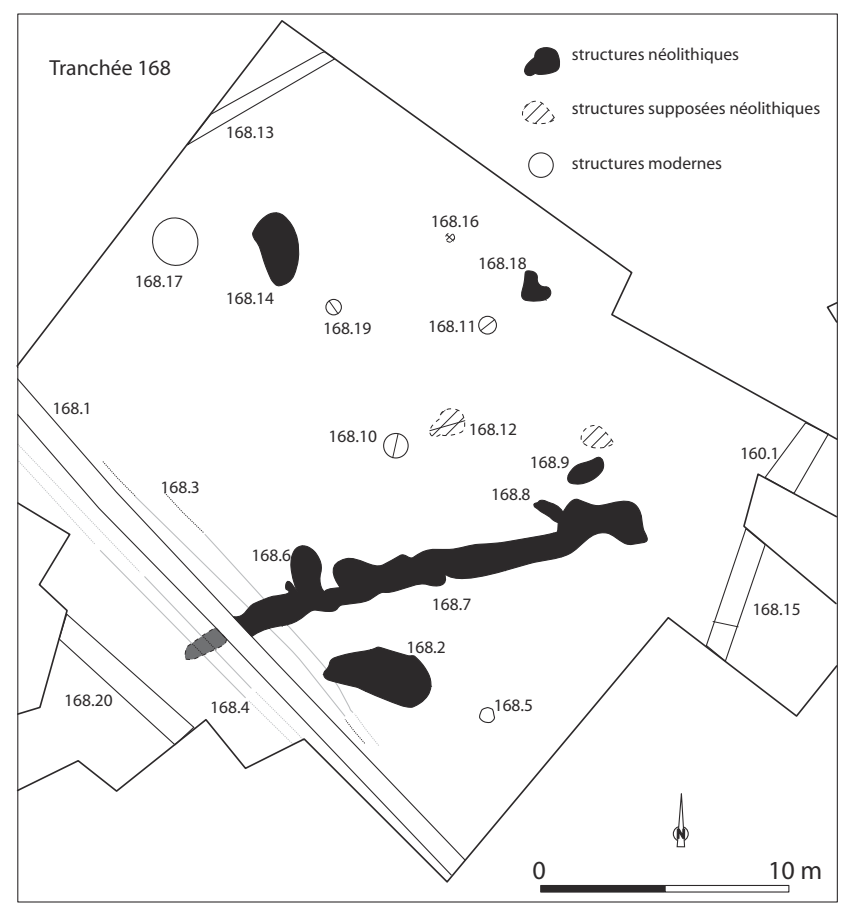

Figure 2 : Vezin-le-Coquet «Les Champs Bleus » : tranche D, plan des vestiges de la tranchée 168. DAO A. Desfonds, V. Brisotto. Figure 2: Vezin-Le-Coquet, "Les Champs Bleus", sector D, plan of the features in trench 168.

\section{La grande fosse F 168.2 (fig. 3)}

Longue de 4,40 mètres et d'une largeur maximale de 2 mètres, cette fosse ovalaire présente des contours plutôt réguliers, avec un léger rétrécissement sur son extrémité ouest. Ses parois sont abruptes à semi-abruptes, et sa profondeur atteint 0,60 mètre. Elle possède un fond relativement plat avec localement des remontées de siltite altérée.

En surface, le sédiment est limono-argileux gris-orangé, parfois beige clair à blanchâtre, assez homogène avec quelques charbons de bois, et quelques fragments de céramique. Un petit niveau rubéfié, situé au centre-ouest de la fosse renfermait des nodules de terre cuite associés à une concentration de charbons de bois. Il pourrait s'agir d'un rejet de foyer.

Les coupes, issues des deux sondages effectués en quinconce, ont permis de mettre en évidence sept US. L'hydromorphie observée dans l'US 5 indique un possible fonctionnement ouvert de cette fosse pendant un laps de temps indéfini. Le prélèvement dans ce niveau n'a malheureusement livré aucun reste pollinique (d'après $\mathrm{D}$. BarbierPain, in Brisotto, 2008).

Le mobilier céramique et lithique se répartit dans les différents niveaux du remplissage mais plus particulièrement 

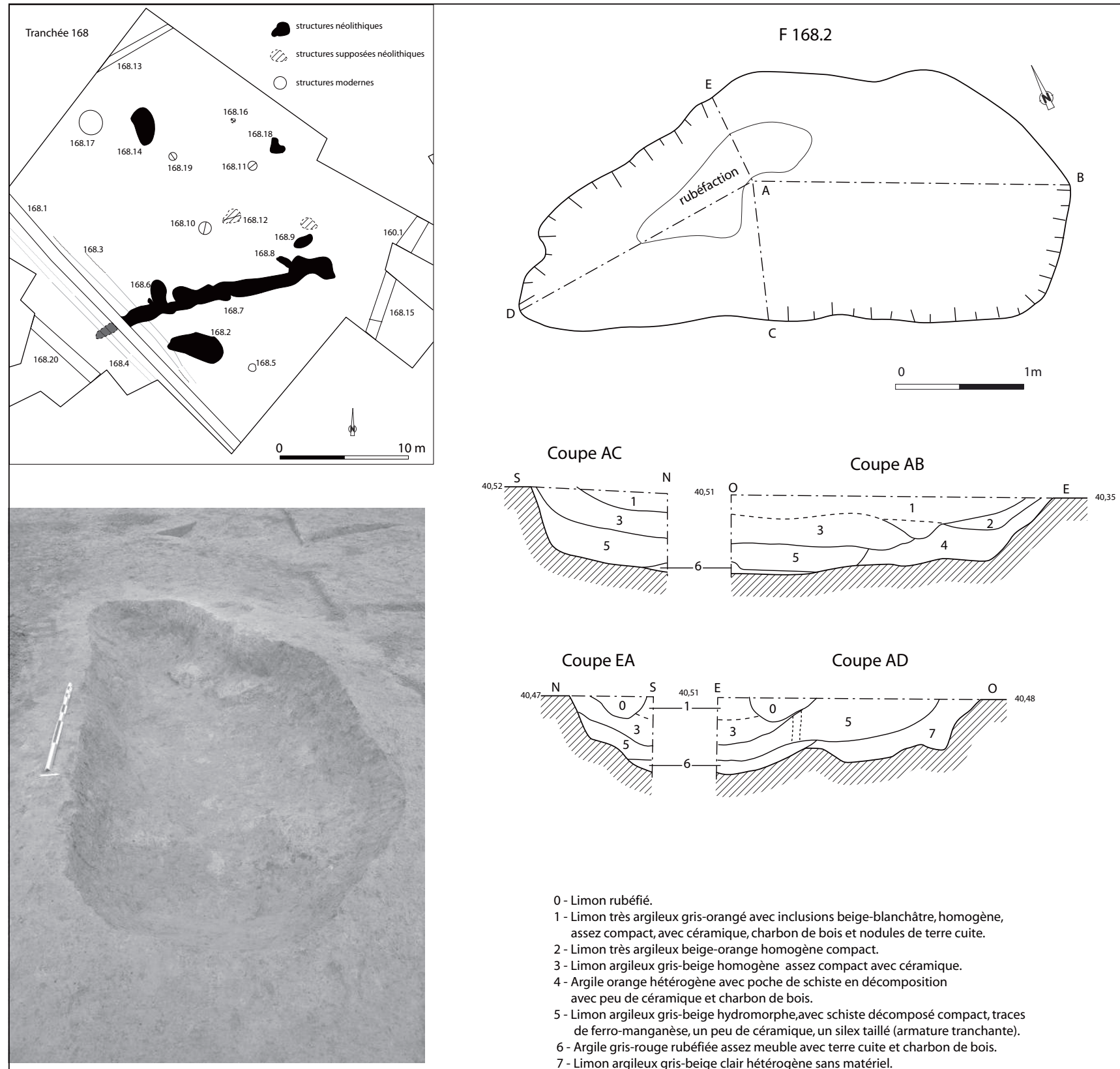

Coupe AD

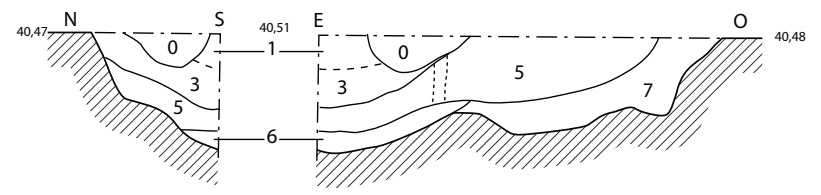

0 - Limon rubéfié.

1 - Limon très argileux gris-orangé avec inclusions beige-blanchâtre, homogène assez compact, avec céramique, charbon de bois et nodules de terre cuite. 2 - Limon très argileux beige-orange homogène compact.

3 - Limon argileux gris-beige homogène assez compact avec céramique.

4 - Argile orange hétérogène avec poche de schiste en décomposition

avec peu de céramique et charbon de bois.

5 - Limon argileux gris-beige hydromorphe,avec schiste décomposé compact, traces

de ferro-manganèse, un peu de céramique, un silex taillé (armature tranchante).

6 - Argile gris-rouge rubéfiée assez meuble avec terre cuite et charbon de bois.

7 - Limon argileux gris-beige clair hétérogène sans matériel.

Figure 3 : Vezin-le-Coquet «Les Champs Bleus » : tranche D, plan et coupe de F 168.2 au 1/50 et photo de la fosse vidée. Relevé S. Bourne, cliché V. Brisotto.

Figure 3: Vezin-Le-Coquet, "Les Champs Bleus", sector D, plan and sections of F $168.21 / 50$ and photo of the excavated ditch.

dans les US 1,3 et 5 . Quatre individus céramiques y ont été individualisés, accompagnés de six pièces lithiques.

On notera également la présence éparse de quelques micro-fragments d'os brûlés, prélevés dans le remplissage. La majorité d'entre eux sont indéterminables. D'autres attestent toutefois de la présence de faune (d'après $M$. Texier, in Brisotto, 2008).
L'éventualité d'une fosse liée à l'extraction, ayant servi par la suite de fosse-rejet reste l'hypothèse la plus probable.

\section{Une structure allongée (F 168.7) : une tranchée de fondation? (fig. 4)}

Le fait 168.7, apparu au premier nettoyage comme plusieurs fosses distinctes, s'est rapidement avéré n'être 


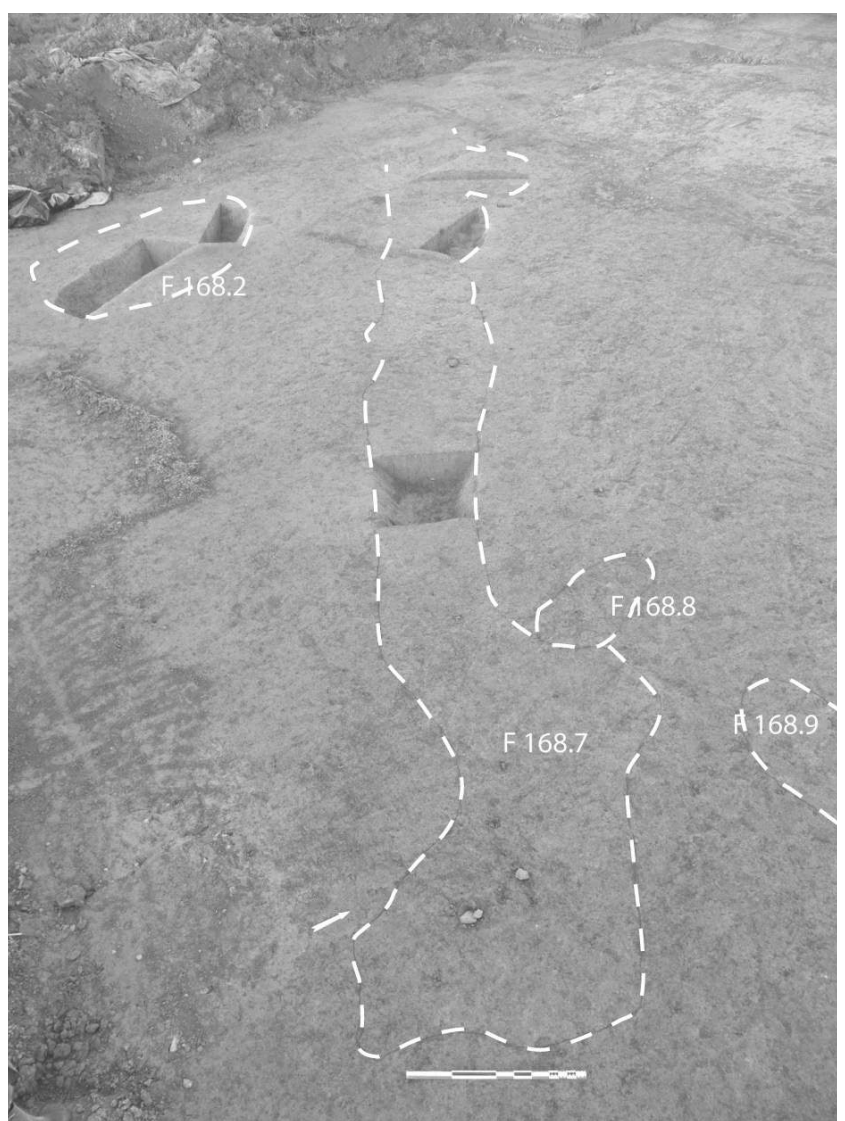

Figure 4 : Vezin-le-Coquet « Les Champs Bleus » : tranche D, vue de la structure allongée F 168.7. Cliché V. Brisotto.

Figure 4: Vezin-Le-Coquet, "Les Champs Bleus", sector D, oval shaped feature F 168.7.

qu'une seule et même structure fossoyée, longue d'environ 20 mètres. Orienté nord-est/sud-ouest, ses contours irréguliers lui confèrent une largeur qui varie de 0,60 mètre à 1 mètre. Alors que l'extrémité nord-est apparaît relativement nette, l'extrémité opposée, au sud-ouest, reste incertaine, du fait de la présence de fossés récents. Les coupes des neufs sondages effectués, ont mis en évidence une à deux US, présentant par endroit de nombreuses bioturbations verticales (fig. 5). La fouille complète de la structure a révélé un profil en cuvette, d'une profondeur oscillant de 0,15 mètre à 0,40 mètre.

Elle a livré une quantité non négligeable de mobilier, localisée essentiellement à son extrémité nord-est, dans les sondages 3 et 4 . La céramique, représentée par cinq individus dont une coupe à socle, reste cohérente avec les éléments issus de la fosse 168.2. Plusieurs éléments lithiques ont également été découverts : une pièce esquillée sur support nucléiforme, une lame de petit module, ainsi qu'une molette en granite, une plaquette de schiste ayant servi d'enclume et une pièce multifonctionnelle façonnée sur dolérite.
L'hypothèse de départ, pressentie sur le terrain, pour cette structure fossoyée longiligne mais irrégulière, plaidait en faveur d'une tranchée liée à une activité d'extraction. Toutefois, au regard d'une largeur et d'une profondeur peu importantes (à moins d'être une tranchée exploratoire), cette proposition a été mise de côté au profit de celle d'une tranchée de fondation. En effet, sa proximité avec la grande fosse-dépotoir d'une part, et un ensemble de petites fosses et trous de poteau d'autre part, suggère la présence d'un bâtiment.

\section{Les fosses et les trous de poteau (fig. 6)}

Le nettoyage fin de la zone a permis de mettre en évidence de petites fosses et trous de poteau, aux contours difficilement lisibles. Leur reconnaissance a été facilitée par la présence d'inclusions beige clair à blanchâtre en surface, dans un sédiment limono-argileux gris-orangé, identique à celui des deux autres faits décrits ci-dessus. La présence de charbons, et de rares tessons de céramique a également servi d'indicateur.

Toutes ces structures se situent au-delà de la tranchée de fondation F. 168.7 dans la partie nord-ouest du décapage.

Deux d'entre elles jouxtent cette dernière (F. 168.6 et F. 168.8). Au total, sept fosses et/ou trous de poteau peuvent, de par leur remplissage ou la présence de mobilier, se rattacher à l'ensemble néolithique.

Leurs morphologies et leurs dimensions sont variables mais leurs remplissages composés d'une à deux unités stratigraphiques sont comparables. Quatre de ces structures ont livré du mobilier, en faible quantité (F. 68.8, F. 168.9, 168.14, 168.18).

Certaines de ces fosses ont dû accueillir des poteaux comme l'atteste les sondages effectués dans les F. 168.12, 168,16 et F. 168.18, qui ont mis évidence des négatifs de poteau et des éléments de calage.

\section{Les autres structures de la tranchée (fig. 2)}

Les quatre fossés, orientés nord-est/sud-ouest (F. 168.1, F. 168.3, F. 168.4 et F. 168.20), sont des fossés parcellaires modernes et contemporains, dont certains figurent sur le cadastre napoléonien. Il en est de même pour le fossé F. 160.1 ou F. 168.15.

Quant aux autres fosses de la zone, (F. 168.5, F. 168.10, F. 168.11, F. 168.17 et F. 168.19), leurs contours réguliers et facilement repérables au décapage, supposaient de ne pas les associer aux structures néolithiques. Les sondages effectués dans plusieurs d'entre elles, ainsi que la présence d'ardoises dans d'autres ont permis de confirmer cette hypothèse. 


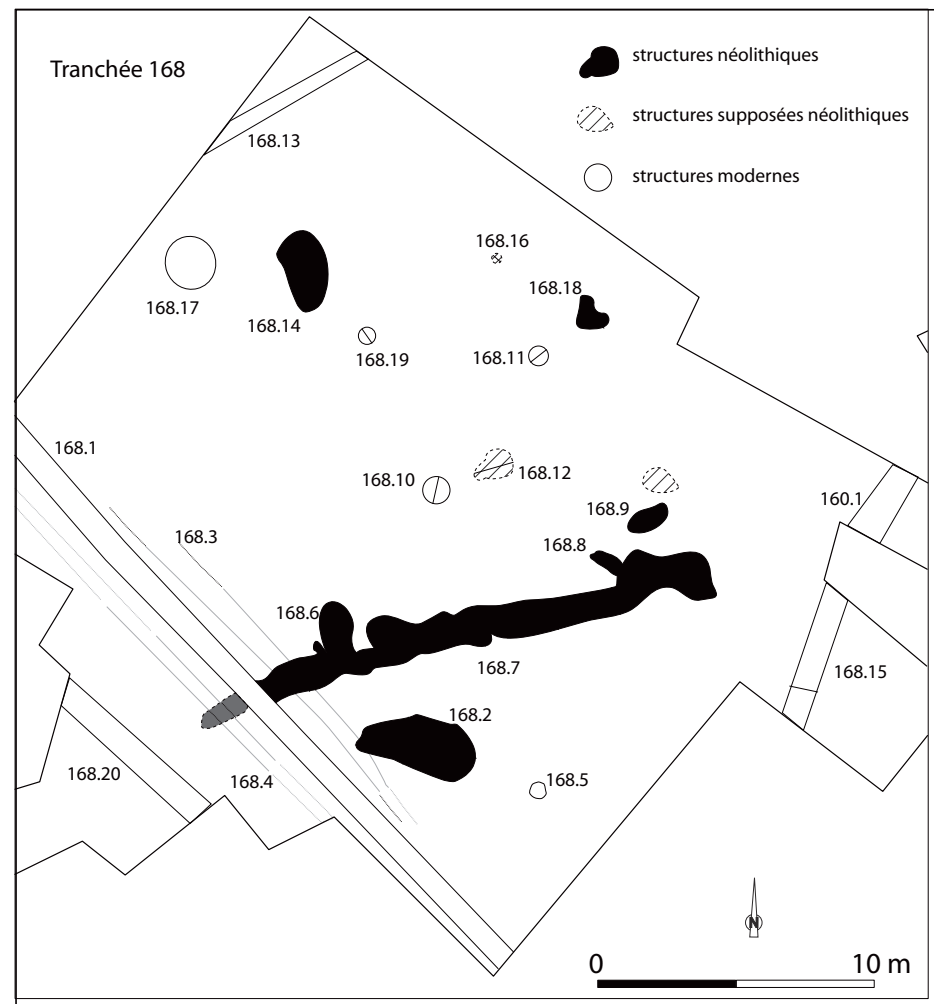

Tranchée 168 - F 7 - sondage 1

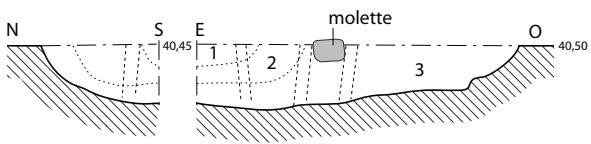

1 - Limon argileux gris-brun sableux.

2 - Limon argileux gris-brun compact moucheté

d' inclusions blanchâtres, traces de ferro-manganèse.

3 - Limon argileux brun orangé compact moucheté

d' inclusions blanchâtres, traces de ferro-manganèse et

graviers de quartz.

Tranchée 168 - F 7 - sondage 2

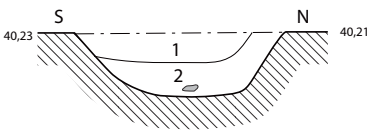

1 - Limon argileux gris-brun orangé compact hétérogène avec inclusions blanchâtres et charbon de bois.

2 - Limon argileux gris-brun orangé meuble homogène avec charbon de bois.

Tranchée 168 - F7 - sondage 3

Tranchée 168 - F 7 - sondage 4
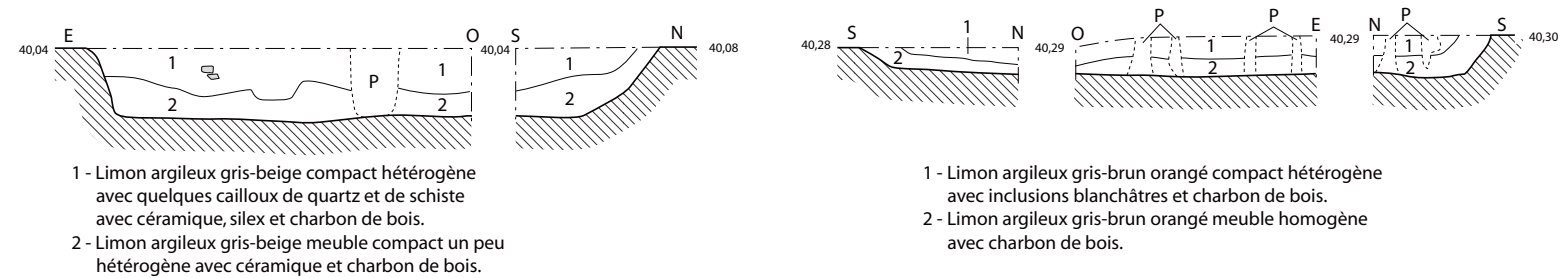

1 - Limon argileux gris-brun orangé compact hétérogène avec inclusions blanchâtres et charbon de bois.

2 - Limon argileux gris-brun orangé meuble homogène avec charbon de bois.

Tranchée 168 - F 7 - sondage 5

Tranchée 168 - F 7 - sondage 6
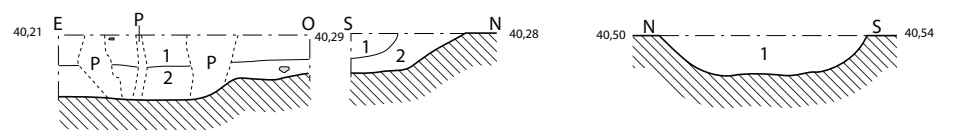

$\mathrm{p}$ : perturbations

1 - Limon argileux gris-brun orangé compact hétérogène avec inclusions blanchâtres et charbon de bois.

2 - Limon argileux gris-brun orangé meuble homogène

1 - Limon argileux brun-gris compact

hétérogène avec nodules gris orangé avec

inclusions blanchâtre, charbon de bois

et ferro-manganèse.

avec charbon de bois.

Tranchée 168 - F 7 - sondage 7

Tranchée 168 - F 7 - sondage 8
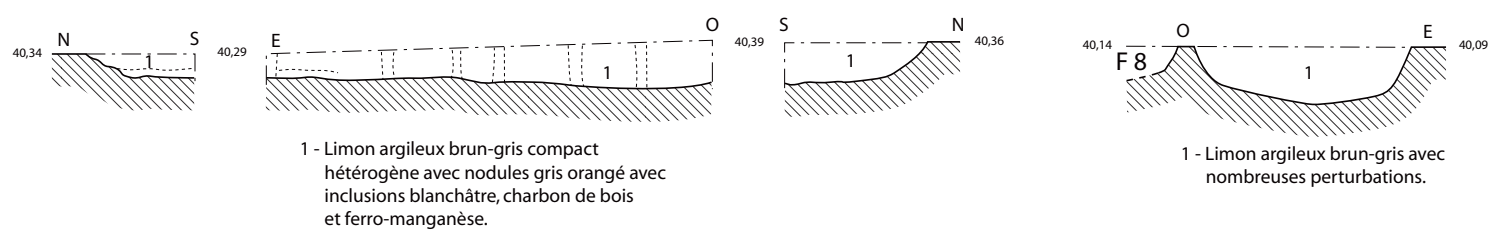

- Limon argileux brun-gris avec
nombreuses perturbations.

inclusions blanchâtre, charbon de bois

Figure 5 : Vezin-le-Coquet " Les Champs Bleus » : tranche D, plan et coupes de F. 168.7. Relevés S. Bourne, V. Brisotto, DAO A. Desfonds, V. Brisotto.

Figure 5: Vezin-Le-Coquet, "Les Champs Bleus", sector D, plan and sections off. 168.7. 


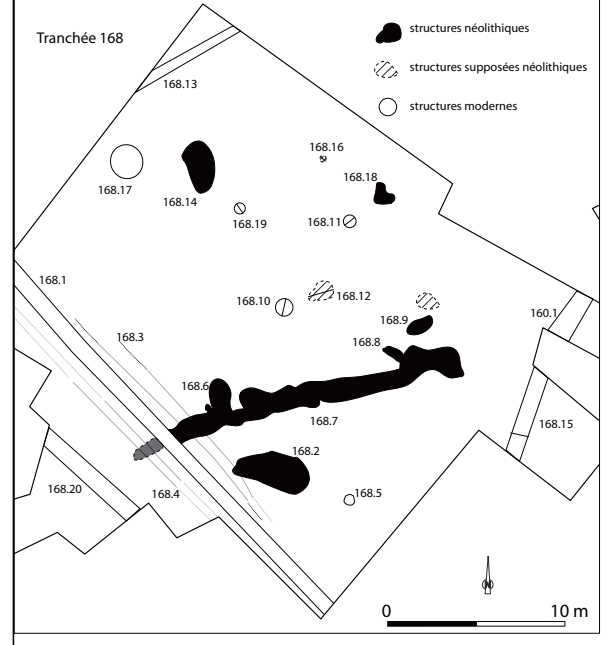

Tranchée 168 - F 8
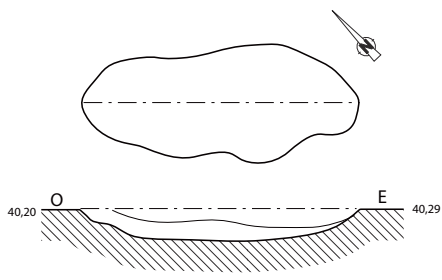

1 - Limon argileux brun-gris compact avec inclusions blanchâtre et ferro-manganèse. 2 - Limon argileux gris-orangé très plastique avec ferro-manganèse.
Tranchée 168 - F 9
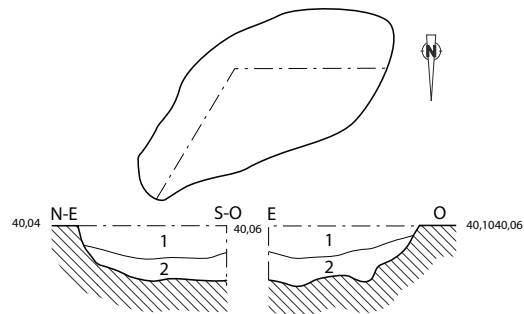

1 - Limon argileux brun-gris hétérogène meuble avec inclusions blanchâtre, céramique et charbon de bois. 2 - Limon argileux orangé compact.

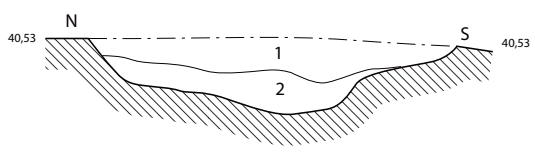

- Limon argileux gris-beige compact homogène avec traces

de ferro-manganèse et charbon de bois.

d' inclusions blanchâtres, de ferro-manogène avec traces

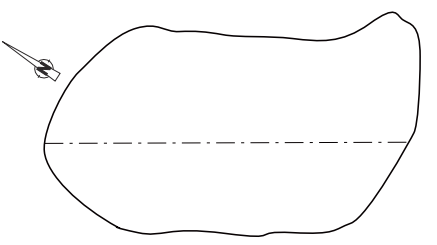

\section{0,53}




\section{Le Mobilier CÉramique (X. HeNaff)}

Le mobilier céramique découvert est partagé entre la tranche 1 (2006) et la tranche D (2008). La première phase n'a livré que quelques tessons hors structure, parmi lesquels un fragment de col décoré et une anse partiellement conservée. En revanche, la deuxième opération a mis au jour, dans la tranchée 168, un ensemble de structures attribuées au Néolithique, qui a livré un total de 271 tessons de céramique. Ceux-ci, après remontage, représentent neuf récipients répartis dans deux structures. Particulièrement fragmenté, ce mobilier se présentait souvent sous forme de concentrations très localisées permettant d'affirmer un écrasement "sur place ", chaque structure ayant livré des remontages de proximité.

\section{Technologie}

Les types de pâtes ne montrent pas de différences notables étant donné que l'épaisseur moyenne des parois $(4-6 \mathrm{~mm})$ et la qualité des individus sont similaires. Pratiquement tous les tessons présentent une assez faible densité d'inclusions (de 5 à $10 \%$ selon l'abaque de Mathew, Woods et Oliver; Orton et al., 1993), associée à une granulométrie fine à moyenne, soit rarement plus de $2 \mathrm{~mm}$ de diamètre.

La technique de montage au colombin a été la seule réellement identifiable sur l'ensemble du corpus. À la faveur de cassures liées au décollement des boudins d'argile, on a relevé un montage en biseau majoritaire. Les traitements de surface indiquent un travail de lissage systématique voire un polissage final. Une cuisson dans un premier temps réductrice semble la méthode la plus communément utilisée : la grande majorité des tessons présente une tranche sombre tandis que les surfaces affichent une large gamme de couleurs qui vont du noir uniforme à l'orange.

\section{Typologie}

\section{Les formes}

Les bols sont, soit à profil légèrement infléchi et à lèvre aplanie (fig. $7, \mathrm{n}^{\circ} 3$; fig. $8, \mathrm{n}^{\circ} 1$ ), soit à profil ovoïde à lèvre en pouce (fig. $\left.7, n^{\circ} 4\right)$. Un individu représente une probable jatte carénée (fig. $7, \mathrm{n}^{\circ}$ 2). Les pots affichent un profil simple (fig. $8, n^{\circ} 2$ ), en $S$ à lèvre amincie (fig. $7, n^{\circ} 5$ ) et caréné (fig. 8, no 4). Un individu correspond à une bouteille (fig. 8, $\mathrm{n}^{\circ} 5$ ). Enfin, une coupe-à-socle à profil concave est présente (fig. 8, no 4).

\section{Les décors}

La totalité de la décoration des vases est réalisée par impression. Il s'agit de l'emploi d'un poinçon de section circulaire ou ovale de 1 à $2 \mathrm{~mm}$ de diamètre enfoncé sur 1 à $2 \mathrm{~mm}$ de profondeur. Les motifs de base sont relativement simples mais donnent naissance à des thèmes quant à eux plus complexes : zigzag, crosse, figure géométrique.

Le bol (fig. 9, $\mathrm{n}^{\circ} 1$ ) porte un décor dont le thème se compose d'une double ligne parallèle de points formant probablement une frise en zigzag.

Le fragment de col provenant de la tranchée 37 (tranche 1, Tournier, 2006) affiche une section d'une ligne courbe de points (fig. $7, \mathrm{n}^{\circ} 1$ ).

La jatte carénée (fig. $7, n^{\circ} 2$ ) arbore un thème composé de trois lignes perpendiculaires formant un rectangle dont la ligne de base est placée juste au-dessus du diamètre maximum. Une partie du décor semble se poursuivre sur la gauche du rectangle en une sorte d'arceau. L'état fragmentaire du vase ne permet pas d'affirmer une continuité, mais il pourrait s'agir d'un décor particulier que l'on qualifie de " corniforme ». Les coups de poinçons sont espacés en moyenne de $2 \mathrm{~mm}$ de centre à centre (fig. 9).

Enfin, la coupe-à-socle (fig. 8, no 4 ) affiche, à quelques centimètres au-dessus de la base du socle, un thème composé d'un champ de poinçons formant triangle pointe en haut.

\section{Attributions chrono-culturelles}

D'un point de vue strictement typologique, les bols et les formes fermées au col resserré et à la panse globuleuse se rencontrent dans les séries de la France du Nord-Ouest à partir de la fin du Néolithique ancien. À l'échelle régionale, des similitudes typologiques se retrouvent dans des séries en contexte d'habitat et funéraire (fig. 10). À proximité de notre site, les fouilles programmées et de sauvetage à Lillemer (Ille-etVilaine) ont livré nombre de formes comparables (Laporte et al., 2003). Dans le domaine ligérien, de nombreux vases provenant du site de Sandun à Guérande (Loire-Atlantique), sont également comparables. En effet, nous pouvons y reconnaître les pots à profil en $S$ et les bouteilles - formes dominantes au sein du corpus (Letterlé et al., 1991; Letterlé, 1997). Bien qu'étant des catégories de forme assez ubiquistes, les bols sont comparables du point de vue technologique (calibre et densité de inclusions; traitement de surface). Enfin, la zone nucléaire Castellic est de loin la plus riche bien que rares soient les sites ayant fourni des corpus conséquents à l'image d'Er-Lannic ou du Lizo dans le Morbihan par exemple (Bailloud, 1975; Le Rouzic, 1930 et 1933). La coupe-à-socle, qui présente un profil concave, appartient à un type de forme assez " classique " et répandu tandis que ses dimensions $(20 \mathrm{~cm}$ de diamètre au bord) la placent dans la catégorie des grands formats (Grouber, 2000). 


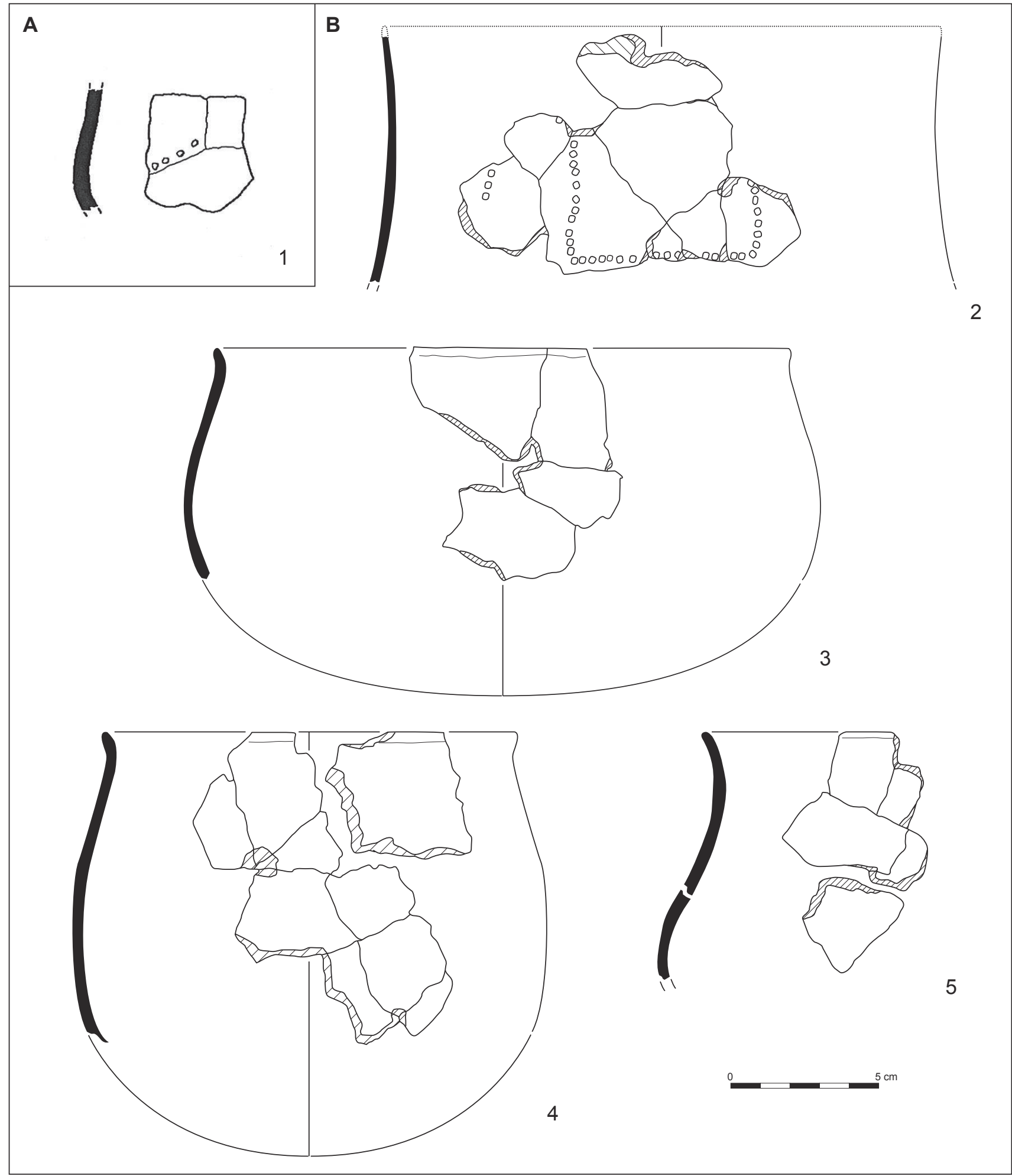

Figure 7 : Vezin-le-Coquet «ZAC des Champs Bleus» (Ille-et-Vilaine). A. : tesson décoré découvert lors du diagnostic de la tranche A (d'après Tournier 2006). B. : mobilier céramique de la structure 2 (tranche D). (C X. Hénaff, Inrap.

Figure 7: Vezin-Le-Coquet, "ZAC des Champs Bleus" (Ille-et-Vilaine). A. Decorated pottery sherd discovered during the first stage of the evaluation (after Tournier 2006). B. pottery from structure 2 (sector D). 

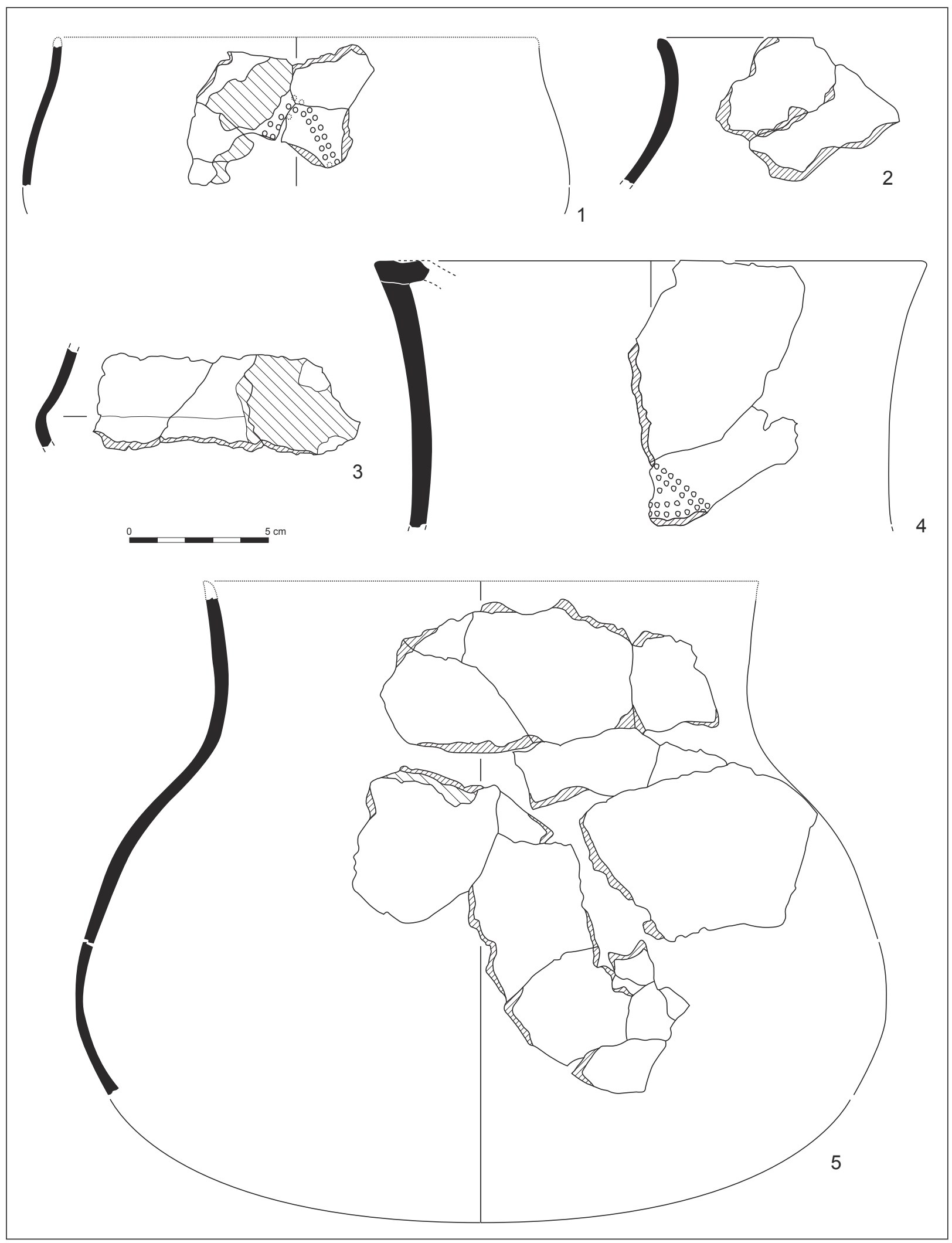

Figure 8 : Vezin-le-Coquet "ZAC des Champs Bleus » (Ille-et-Vilaine). Mobilier céramique de la structure 7 (tranche D). N ${ }^{\text {os }} 1-4$ : sondage $3 ; n^{\circ} 5$ : sondage 8 . (C) X. Hénaff, Inrap.

Figure 8: Vezin-Le-Coquet, "ZAC des Champs Bleus" (Ille-et-Vilaine). Pottery from structure 7 (sector D). $N^{\circ}$ 1-4: trench 3; $n{ }^{\circ} 5$ : trench 8. 


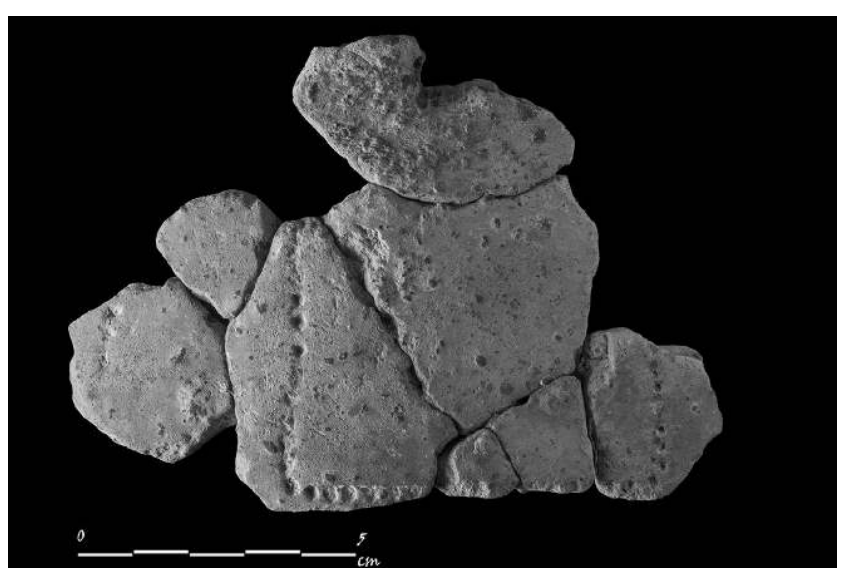

Figure 9 : Vezin-le-Coquet "ZAC des Champs Bleus » (Ille-etVilaine). Photographie de détail du décor « corniforme » du vase provenant de la structure 2. (C) H. Paitier, Inrap.

Figure 9: Vezin-Le-Coquet, "ZAC des Champs Bleus" (Ille-et-Vilaine). Detail of the "Horn motif" décor from structure 2.

Les thèmes décoratifs présents à Vezin-le-Coquet ne sont pas sans rappeler les séries de style Castellic.

Le premier thème reconnu sur le vase provenant du Fait 2 est très particulier et renvoie sans conteste à l'art mégalithique morbihannais (fig. 11). Très fréquent, le signe en $\mathrm{U}$ aux branches plus ou moins rabattues vers l'extérieur - appelé le « juguiforme " puis le " corniforme/cornu " apparaît sur divers monuments, tantôt très anciens comme à Barnenez dans le Finistère (Giot, 1987), tantôt plus tardifs comme à Gavrinis dans le Morbihan (Le Roux, 2006). Ce signe peut être représenté isolé, associé à d'autres signes comme l'« écusson " du Mané-er-Hroeg à Locmariaquer (Mens, 2006), ou bien multiplié en séries emboîtées ou juxtaposées sur une même surface comme sur plusieurs stèles du dolmen du Mané-Lud à Locmariaquer (Cassen, 2007), pour ne citer que ces exemples. En revanche, le « corniforme " n'est présent, à notre connaissance, que sur un vase découvert dans le coffre supérieur du tertre détruit de Saint-Germain-en-Erdeven (Le Boulaire et Cassen, 2000). En outre, ce signe a longtemps été interprété comme la représentation stylisée d'un crâne de taureau avec ses cornes (Cassen et L'Helgouac'h, 1990), mais les dernières études réalisées semblent contester la première version pour aller dans le sens d'un oiseau en vol (Cassen, 2007, 2011).

Le thème du zigzag présent sur le bol provenant du Fait 7 est beaucoup plus répandu. En effet, nous le retrouvons sur nombre de vases provenant de plusieurs dolmens morbihannais, comme au tertre détruit de Saint-Germain-en-Erdeven par exemple (Le Boulaire et Cassen, 2000), ou sur les sites du Lann Vras au Castellic et d'Er-Lannic (Bailloud, 1975). Cependant, la technique de la cannelure est la plus usitée, contrairement à celle du point présente à Vezin-le-Coquet.
On remarque par ailleurs que cette technique est surtout présente sur les coupes-à-socle (Grouber, 2000).

Le dernier thème présent sur la coupe-à-socle provenant également du Fait 7 de Vezin-le-Coquet, est entièrement réalisé au point. Ce thème correspond à une figuration très courante sur ce type de support. En effet, nombre d'individus présents à Er-Lannic ou à Locmariaquer dans le Morbihan livrent des thèmes identiques centrés sur des formes géométriques dérivées du triangle notamment (Grouber, 2000; Le Roux et al., 2006).

Malgré sa faible quantité, le matériel céramique de Vezin-le-Coquet évoque par ses caractères technologique et typologique mis en évidence un assemblage attribuable au Néolithique moyen armoricain. La présence d'une coupeà-socle associée à la faible richesse décorative du mobilier (motifs et compositions moins variés; aucun bouton au repoussé, ni cannelure) permet de proposer une attribution à la phase récente du faciès Castellic (Cassen, 2000) dont la présence sur tout le Massif armoricain n'est plus à démontrer (Cassen et al., 2000; Hamon, 2003).

La petite occupation de Vezin-le-Coquet permet donc d'apporter un élément supplémentaire non négligeable dans la mesure où cette région, située entre la zone nucléaire carnacoise et la côte septentrionale de la Bretagne, n’a livré que très peu d'éléments de style Castellic à ce jour (fig. 12).

\section{Le MOBILIER LITHIQUe (S. BOURNe)}

Le mobilier lithique taillé provenant des structures en creux néolithiques (tranchée 168) est composé d'un ensemble restreint de onze pièces. Six pièces proviennent du Fait 168.2 (US 1, 3, 5 et 6), une du Fait 168.6, et quatre du Fait 168.7. Ce petit lot est complété par vingt-deux pièces découvertes en périphérie du site, lors des trois opérations de diagnostic qui se sont succédées sur la ZAC « les Champs Bleus » (Tournier, 2006; Brisotto, 2011 et 2008). Pour certaines d'entre elles, au vu de leur distribution générale à proximité de la tranchée 168, l'hypothèse de leur contemporanéité avec l'occupation du Néolithique moyen II peut être formulée. C'est plus particulièrement le cas pour les trois pièces découvertes en 2006. L'une d'elles, située à l'ouest, et à cent mètres seulement de la concentration de structures néolithiques, est un grattoir sur éclat laminaire. Elle est associée à un fragment de céramique fine décorée (Tournier, 2006), caractéristique de la phase récente du Castellic (fig. 7 , $\left.\mathrm{n}^{\circ} 1\right)$.

Une autre concentration de pièces lithiques taillées, située à quatre cents mètres au nord-est de la tranchée 168 a été repérée au cours de la troisième opération (tranches $\mathrm{B}$ et $\mathrm{C}$ ). Ce petit ensemble de dix pièces a été récolté hors structure, 


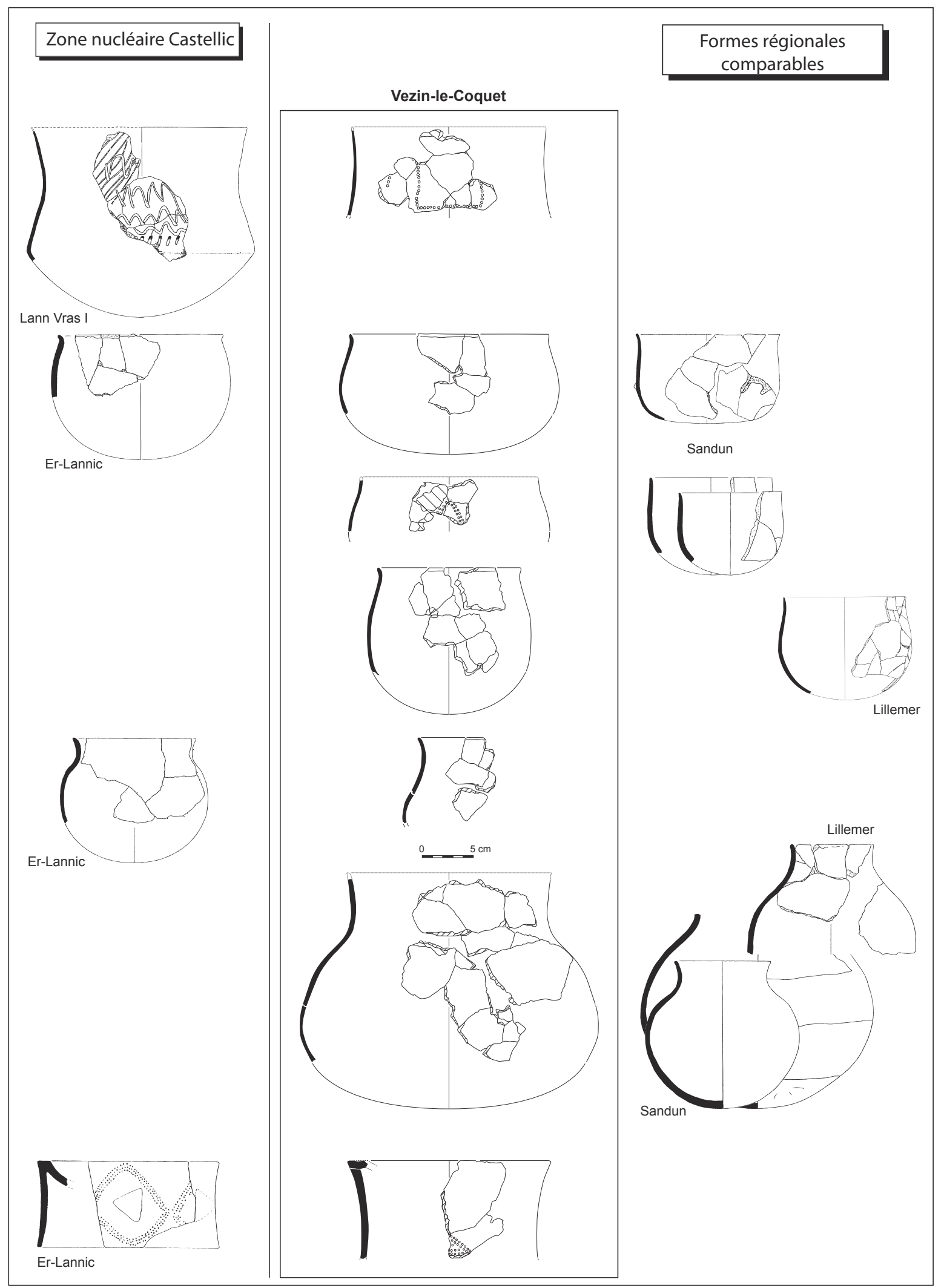

Figure 10 : Correspondances typologiques entre la production céramique régionale et celle de Vezin-le-Coquet (d'après Bailloud, 1975; Letterlé, 1997; Grouber, 2000 ; Hamon G., 2003, Laporte et al., 2003). C X. Hénaff, Inrap.

Figure 10: Typological correspondences between regional pottery production and that of Vezin-le-Coquet. 


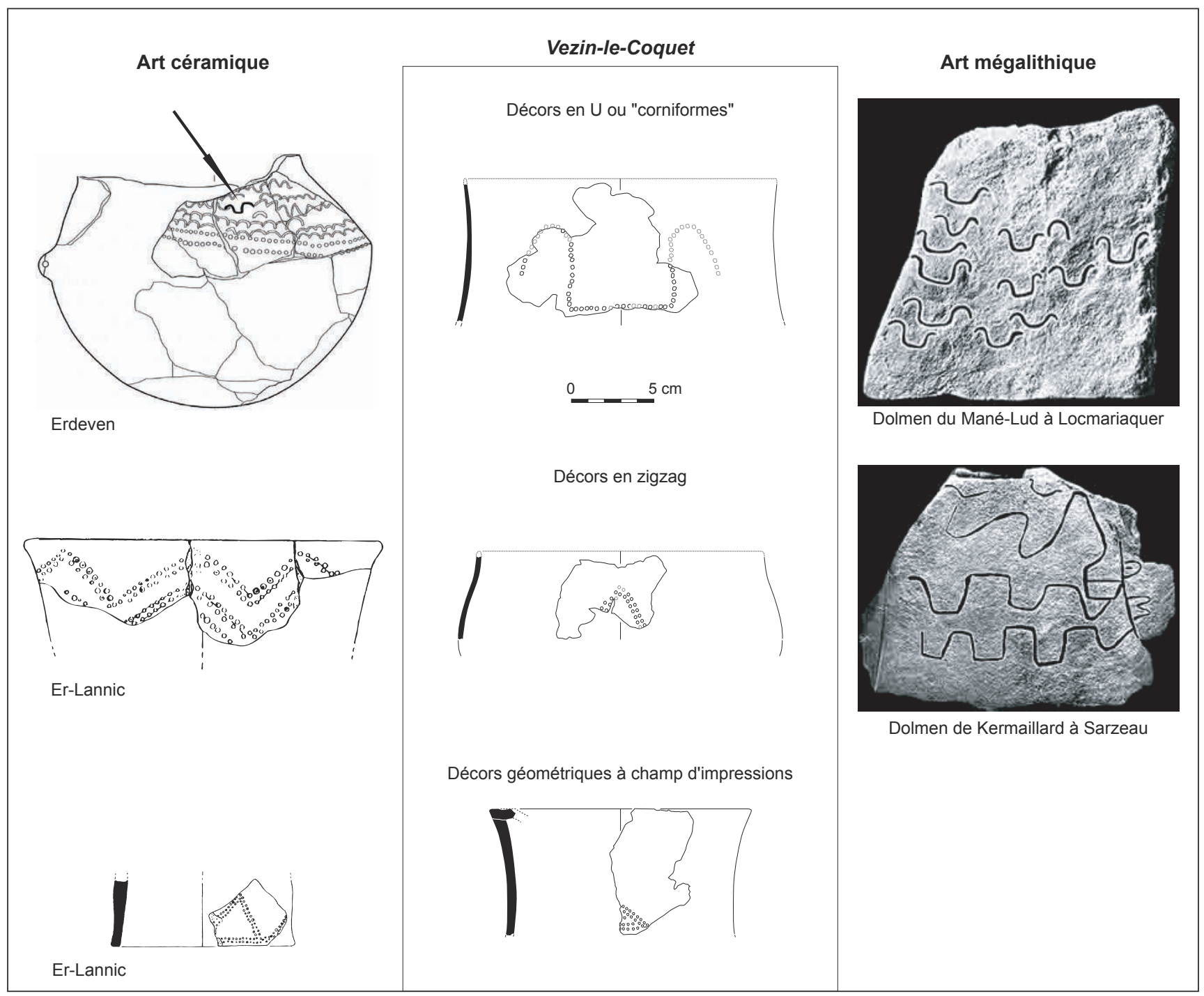

Figure 11 : Correspondances typologiques entre les thèmes décoratifs Castellic et ceux de Vezin-le-Coquet (d'après Bailloud, 1975, Grouber, 2000, Le Boulaire et Cassen, 2000). (C X. Hénaff, Inrap.

Figure 11: Typological correspondences between the Castellic decorative themes and those of Vezin-le-Coquet.

dans un niveau de colluvion, en contrebas de deux structures à pierres chauffées (Brisotto, 2011).

\section{Matières premières}

La diversité des matières premières utilisées pose la question du territoire d'approvisionnement en roches siliceuses des occupants du site. Le bassin sédimentaire de Rennes, et plus généralement tout le Massif armoricain est pauvre en ressource minérale siliceuse, ce qui a conduit les populations locales du Néolithique à varier leurs sources d'approvisionnement, et à trouver sur place des matériaux dits de "substitution» (Dabard et al., 2012). Lidentification des origines géologiques des matières siliceuses permet d'appréhender les territoires parcourus par les populations du Néolithique, ainsi que les contacts existant entre des différents groupes culturels contemporains. Ainsi, les problématiques liées aux sources d'approvisionnement en silex dans le Massif armoricain ont été développées de longue date. C'est sur la base de travaux récents (Ihuel, 2004 et 2012; Dabard et al., 2012; Forré in Blanchet, 2007), ainsi que sur les observations de G. Marchand, qu'au moins cinq types de matières premières ont pu être reconnues dans le petit assemblage lithique récolté à Vezin-le-Coquet.

La matière première la plus représentée (dix pièces dont trois dans le comblement des structures néolithiques) est un 


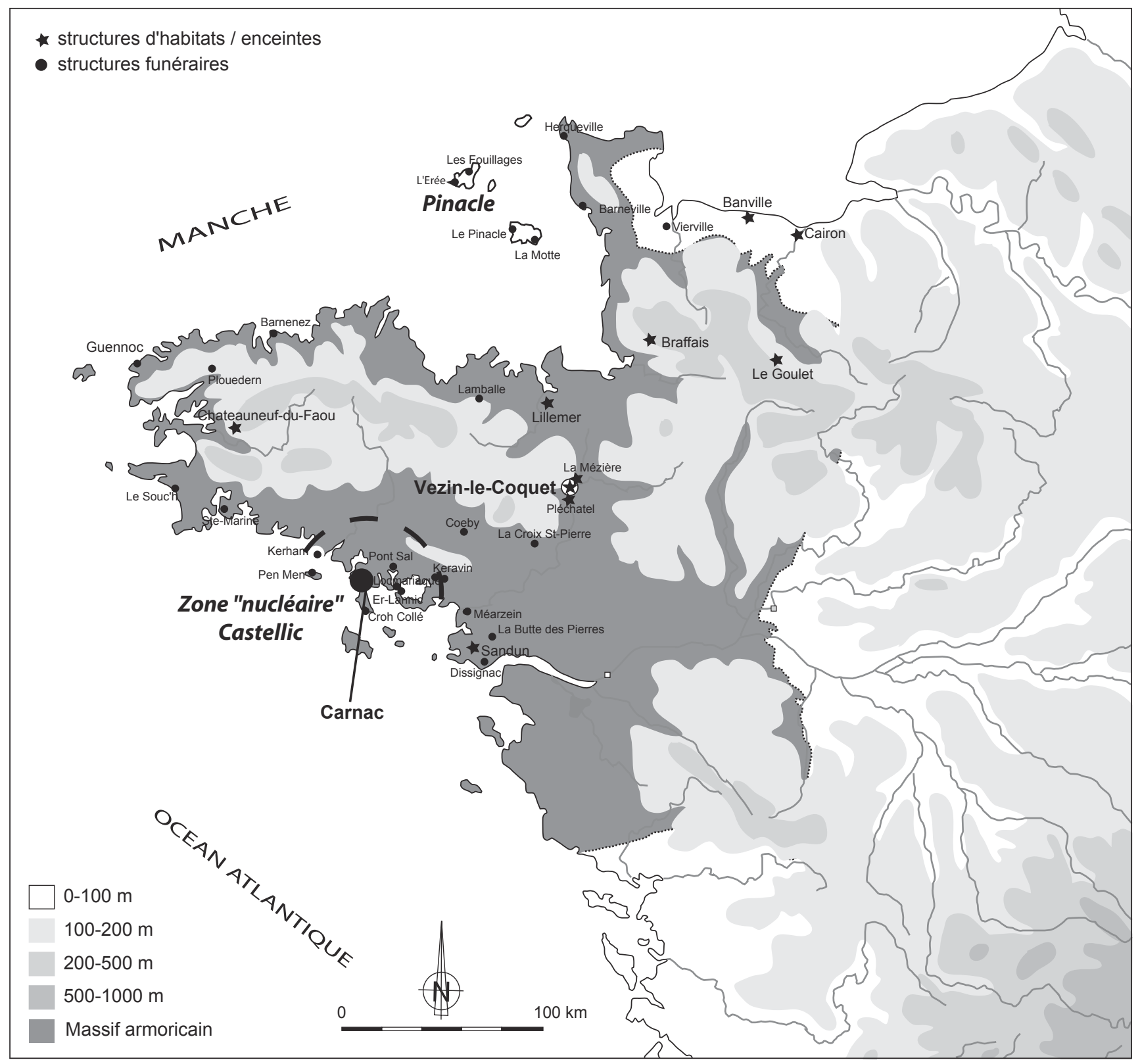

Figure 12 : Localisation des principaux gisements de tradition Castellic et contemporains de l'Ouest de la France. (C) X. Hénaff, Inrap. Figure 12: Location of the main Castellic sites and other contemporary sites in Western France.

silex crétacé blond translucide et brillant, à grain très fin, à trame uniforme et texture homogène. Ce silex est probablement issu des niveaux du Turonien inférieur qui affleurent dans les vallées du Cher et du Loir. On retrouve ce même silex en position secondaire dans les alluvions de la Loire.

D’autres silex du Crétacé (Cénomanien et Sénoniens) sont présents dans la série (sept pièces dont deux dans le comblement des structures néolithiques). Il s'agit de silex marron foncé à noir, brillant et opaque, à grain fin, de texture homogène et de trame uniforme. Les gîtes primaires de ces silex se situent principalement dans le Bassin parisien, en marge orientale du Massif armoricain, mais les caractères ubiquistes de ses multiples faciès permettent difficilement de les rapprocher d'un gîte précis. Ces silex se retrouvent également en position secondaire sous forme de galets côtiers sur les cordons littoraux de la Manche et dans les dépôts alluviaux de la Loire, mais il ne semble pas que ces galets provenant des gîtes secondaires aient été utilisés à Vezin-le-Coquet du fait de l'absence totale de cortex alluvial ou marin sur les pièces observées. 
Le silex jurassique (Bathonien/Bajocien) est représenté par un unique éclat, trouvé dans le comblement du fossé 168.7. Ce silex, d'excellente qualité pour la taille, est marron, à structure très homogène et grain fin. Les affleurements de ce silex se situent au sud de la plaine de Caen et d'Argenton (Calvados), à plus de deux cents kilomètres de Vezinle-Coquet.

L'opale résinite correspond à une silicification tertiaire (Eocène - Bartonien). Elle se caractérise par une surface patinée blanche unie, son grain est très fin. Les sources d'approvisionnement connues les plus proches sont situées dans le Saumurois à environ cent vingt kilomètres de Vezinle-Coquet. Au total, cinq pièces en opale résinite ont été récoltées sur l'emprise des trois diagnostics, dont trois pièces dans le comblement des structures néolithiques.

Enfin, quatre éclats de grès lustré proviennent de la tranchée 110, sur l'emprise de la tranche B du diagnostic. La provenance de ce grès, sans doute local, n'a pas pu être déterminée.

\section{Aspects technologiques et typologie}

Les quelques pièces découvertes à Vezin-le-Coquet ne permettent pas de tirer des informations précises quant aux modalités de débitage ou aux objectifs de la production. Mise à part un unique élément débité au percuteur tendre, la percussion directe à la pierre dure reste la norme, comme l'indique la combinaison de talons larges, de cônes incipients et de bulbes proéminents parfois élidés par des esquilles bulbaires.

L'outillage récolté comporte huit pièces. Quatre d'entre elles ont été découvertes dans le comblement des structures néolithiques de la tranchée 168 : une armature tranchante, un outil multiple, une lame retouchée et une pièce esquillée.

L'outil multiple, dont le support est un éclat en silex blond, présente une retouche directe et une coche sur le bord droit. Le bord proximal, correspondant à une cassure par flexion, est repris par une retouche marginale inverse (fig. 13, $\mathrm{n}^{\circ}$ 5). La lame de section triangulaire est en silex blond du Turonien inférieur. Elle présente une retouche marginale et discontinue sur ses deux bords (fig. 13, $\mathrm{n}^{\circ} 3$ ). La pièce esquillée est réalisée sur un support nucléiforme en silex blond (fig. 13, n 4). L'armature tranchante découverte dans l'US 5 de F. 168.2 est de forme trapézoïdale. Elle a été réalisée sur un support laminaire à trois pans. Le bord droit est rectiligne, il est repris par une retouche directe abrupte et continue, alors que le bord gauche montre une délinéation peu régulière, formée par une retouche inverse et semi-abrupte. Le silex (crétacé) est de couleur brun-gris, d'aspect peu homogène, brillant et opaque. Son grain est fin à trame non uniforme et marbrée d'inclusions blanches assez nombreuses (fig. 13, $\mathrm{n}^{\circ} 2$ ).

Les six autres pièces retouchées ont été trouvées disséminées sur l'emprise des trois diagnostics. Cet ensemble comporte une coche, une pièce esquillée, une lame en silex blond $\mathrm{du}$ Turonien inférieur à retouche d'utilisation et lustré, un grattoir sur éclat laminaire en silex beige, un fragment d'outil indéterminé et une armature tranchante.

Cette deuxième armature tranchante, découverte en 2011, est également de forme trapézoïdale. Réalisée sur un segment mésial de lame de section irrégulière en silex brun crétacé, elle est formée par une retouche directe abrupte et continue sur ses deux bords (fig. 13, n 1 ).

\section{Comparaisons}

Le site de Vezin-le-Coquet s'inscrit dans un réseau d'approvisionnement en matières premières siliceuses qui semble principalement axé vers le sud-est (vallées de la Loire et du Cher, Saumurois), même si quelques éléments indiquent également un approvisionnement vers les marges orientales du Massif armoricain (Normandie, voire Bassin parisien).

L'absence de silex issus de galets côtiers, bien qu'à pondérer du fait de l'indigence de la série, semble être un des caractères spécifiques de l'assemblage présenté ici. Les séries lithiques locales bien documentées montrent toujours une part importante de cette industrie sur galets côtiers $(5,8 \%$ du corpus du site de Lillemer; Guyodo, 2001).

Le caractère prédominant du silex blond du Turonien inférieur semble être l'autre spécificité de la série. Cette matière première est souvent minoritaire, voire anecdotique dans les séries lithiques locales datant du Néolithique moyen, comme à Lillemer où elle est représentée par seulement trois pièces (Guyodo, 2001). En revanche, si l'on se tourne vers le golfe du Morbihan et le sud de la Bretagne, le silex blond du Turonien inférieur est toujours présent sous forme de produits débités, comme aux dolmens de Kerhan 1 et 2 (Saint-Philibert, 56; Guyodo, 2001). On le retrouve également, dans de faibles proportions, sous forme de dépôts de grandes lames, tant en contexte d'habitat (Sandun, 44; Gourio, 2008) qu'en contexte funéraire (La Table des Marchands, Locmariaquer, 56; Le Roux et al., 2006). Au vu de l'absence de cortex alluvial sur les éléments en silex $\mathrm{du}$ Turonien inférieur, il ne semble pas que cette matière première ait été collectée dans les alluvions de la Loire, mais plutôt directement sur les gîtes primaires comme l'indiqueraient les quelques zones corticales, crayeuses et non roulées, conservées sur certaines de ces pièces.

Mais la question de la représentativité de la série lithique mise au jour à Vezin-le-Coquet se pose et nous oblige à nuancer ces constats; l'absence de cortex roulé sur les pièces 


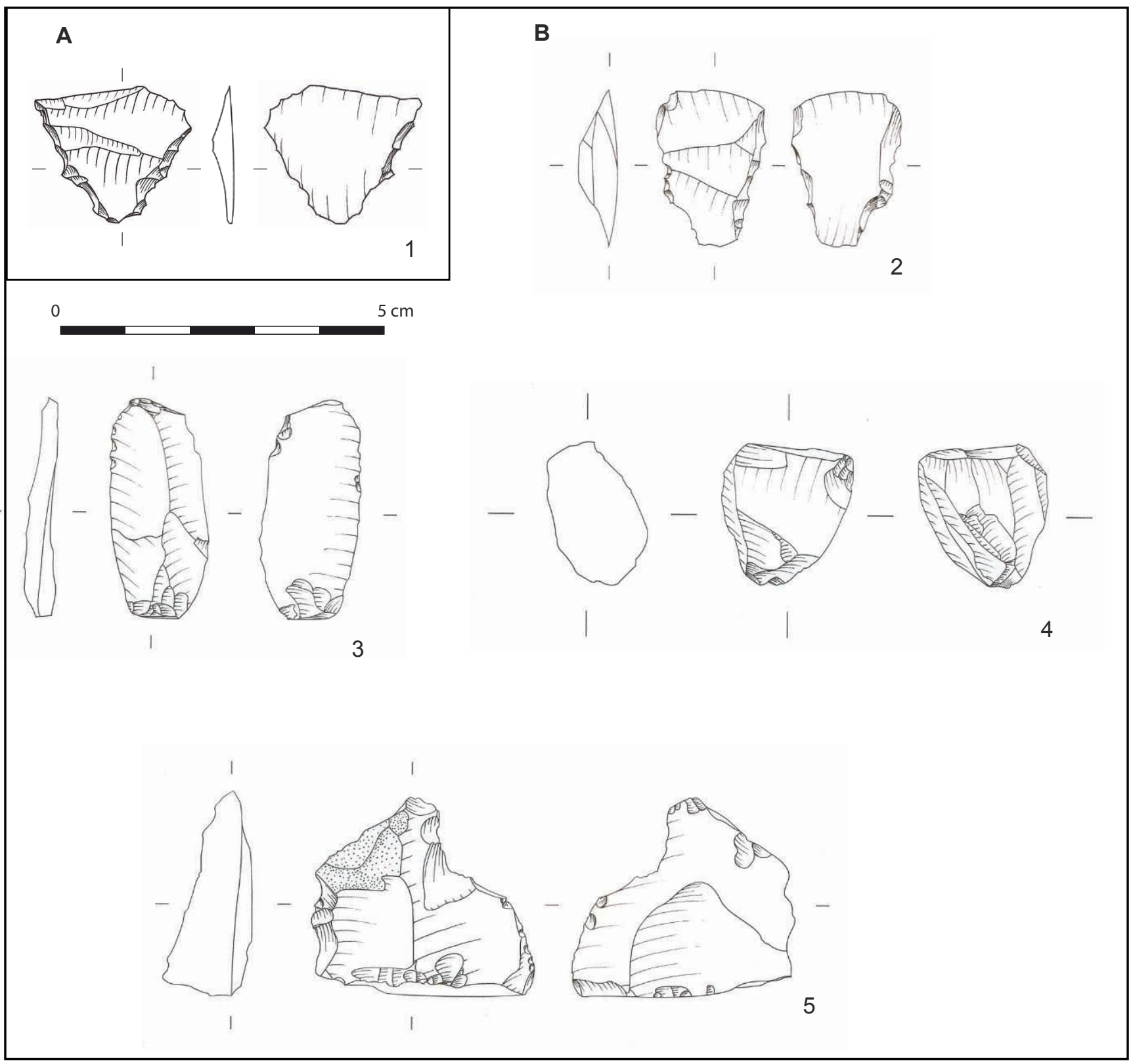

Figure 13 : Vezin-le-Coquet "ZAC des Champs Bleus». A. : armature trapézoïdale issue du diagnostic des tranches B et C, 2011. B. : pièces lithiques provenant des structures néolithiques de la tranchée 168 (tranche D). (C) S. Bourne, Inrap.

Figure 13: Vezin-Le-Coquet, "ZAC des Champs Bleus" (Ille-et-Vilaine). A. trapezoidal arrowhead from sector B and C, 2011. B. Flint from the Neolithic structures of trench 168 (sector D).

observées, tout comme la proportion importante de silex blond ne reflète peut-être pas la réalité du fait de l'indigence de la série.

La présence d'opale résinite ne semble pas aller a contrario de ce que l'on connaît dans les séries lithiques bretonnes. Cette matière première prend toujours part dans des proportions variées dans les assemblages lithiques. Pour le Néolithique ancien, le site du Haut Mée à Saint-Étienneen-Coglès (35) a livré trois éclats d'opale résinite (Cassen $e t$ al., 1998). L'habitat de Pluvignon à Betton (35) a livré une proportion bien plus importante de différentes silicifications tertiaires $(18,17 \%$ de la masse totale), dont de l'opale résinite (Blanchet, 2007). Au Néolithique moyen, son utilisation est toujours bien attestée, comme sur le site du Bois à Pléchatel (Aubry, 2011; Juhel, en cours), aux Lignes de la Gonzée à la Mézière (Escats, 2011; Blanchet, en cours), ou à Lillemer, où elle représente 3,3\% de la série (Guyodo, 2001). 
Les sites d'habitat du Néolithique moyen II bien documentés sont rares en Bretagne. Le site de Lillemer (35) (Guyodo, 2001) reste pour l'instant la seule référence publiée pour cette période dans l'Ille-et-Vilaine. La série lithique issue de ce site montre un débitage d'éclats par percussion directe dure, supports de grattoirs, perçoirs, coches et pièces esquillées. Quant au débitage laminaire, il reste minoritaire. Il est essentiellement effectué au percuteur dur. Les supports laminaires sont réservés aux armatures tranchantes, couteaux, troncatures et burins. Les armatures tranchantes sont majoritairement trapézoïdales, plus rarement triangulaires, sur fragments mésiaux de lames peu larges $(20 \mathrm{~mm}$ en moyenne), formées par une retouche directe abrupte (et très rarement inverse).

Les quelques éléments observés à Vezin-le-Coquet, tant d'un point de vue technologique que de l'assemblage typologique ne semblent pas aller à l'encontre des observations faites à Lillemer (Guyodo, 2001), et les deux armatures s'intègrent bien dans cet horizon du Néolithique moyen II (Marcigny et al., 2010).

\section{L'outillage MaCro-lithiQue (V. BRISOTto)}

L'outillage macrolithique issu de l'occupation néolithique est représenté par trois individus provenant de la structure 168.7 (fig. 14), auxquels s'ajoutent deux plaques de schiste d'une trentaine de centimètres de long, prélevées dans la fosse F. 168.2. L'une d'elles semble porter des traces diffuses de façonnage et/ou d'utilisation opportuniste.

Concernant l'outillage, la première pièce (fig. 14, $\mathrm{n}^{\circ} 1$ ), localisée à la surface du sondage 1 (structure 168.7), est une molette façonnée dans un granite à deux micas, à grains moyens. Longue de $18 \mathrm{~cm}$ pour une largeur de $13 \mathrm{~cm}$, son épaisseur maximum est de $5,5 \mathrm{~cm}$. Les flancs de l'objet ont été épannelés, et/ou bouchardés, alors que le dos plutôt irrégulier, présente une petite surface polie légèrement concave, résultant probablement d'une première vie de l'objet comme meule ou molette. À l'opposé, la face active est planoconcave longitudinalement, et plano-convexe transversalement. Elle présente de larges plages polies, voire lustrées, localisées surtout en périphérie de la surface. Le centre de la pièce, où l'usure est moins marquée, laisse percevoir des traces de piquetage.

Le même sondage a livré une petite plaquette de schiste local (fig. 14, $\mathrm{n}^{\circ} 3$ ), peu épaisse, de forme triangulaire, avec une légère dépression circulaire présentant des traces de percussion et d'écrasement. Il pourrait s'agir d'une petite enclume ou d'une pierre à concasser.

La dernière pièce, provenant du sondage 8 (fig. 15, no 2), est un outil multifonctionnel sur dalle de dolérite. L'une de ses faces ayant servie de molette, présente des plages polies, localisées essentiellement à la périphérie de la surface. Les traces de piquetages, visibles dans la partie centrale, pourraient correspondre à l'entretien de la surface active, mais certaines, plus profondes, évoquent une utilisation postérieure, sans doute comme enclume. La face opposée présente en son centre une large zone circulaire, finement piquetée. Ces nombreux petits impacts, creusant légèrement la surface, suggère ici une utilisation d'enclume. Enfin, l'une des tranches, présente une arête vive, dégageant une sorte de tranchant, marqué par quelques petits négatifs d'enlèvements.

Ce petit ensemble de macro-outillage révèle, en premier lieu, un apport extra local de matières premières comme le granite et la dolérite.

Par ailleurs, il atteste également d'activités domestiques, pouvant s'accorder avec l'hypothèse d'un habitat.

\section{Les datations absolues (TABl. 1)}

Quatre échantillons de charbons de bois, prélevés dans trois structures ayant livré du mobilier néolithique F. 168.2, F. 168.7 et F. 168.9 - ont été soumis à une analyse radiocarbone, par le biais du SRA de Bretagne, dans le cadre de la programmation nationale Artemis (tabl. 1).

Les analyses ont été réalisées par accélérateur au centre de datation par le radiocarbone de Lyon. Les résultats obtenus pour les Faits 168.7 (4459 à 4346 av. J.-C.) et 168.9 (4315 et 4045 av. J.-C.) apparaissent cohérents avec l'assemblage céramique et lithique renvoyant à la sphère Castellic du Néolithique moyen régional.

En revanche les deux échantillons de la grande fosse F2 (US 5 : 4719- 4545 av. J.-C., et US 1 : 3353-3028 av. J.-C.) affichent des datations qui non seulement offrent une amplitude importante entre eux mais également qui ne concordent pas avec l'étude de la céramique. Le charbon issu du comblement terminal renvoie à un Néolithique récent alors que le fond du creusement se situe entre la fin du Néolithique ancien et le début du Néolithique moyen I.

Si l'on ne peut exclure une possible pollution ou le fait d'une perturbation, notamment pour le charbon issu du niveau de surface, l'éventualité d'une occupation plus précoce peut être envisagée.

\section{ESSAI DE COMPARAISON ET D'INTERPRÉTATION}

À l'échelle régionale, les occupations relatives aux productions céramiques Castellic sont essentiellement connues dans la zone nucléaire du sud Morbihan, notamment sur les sites 

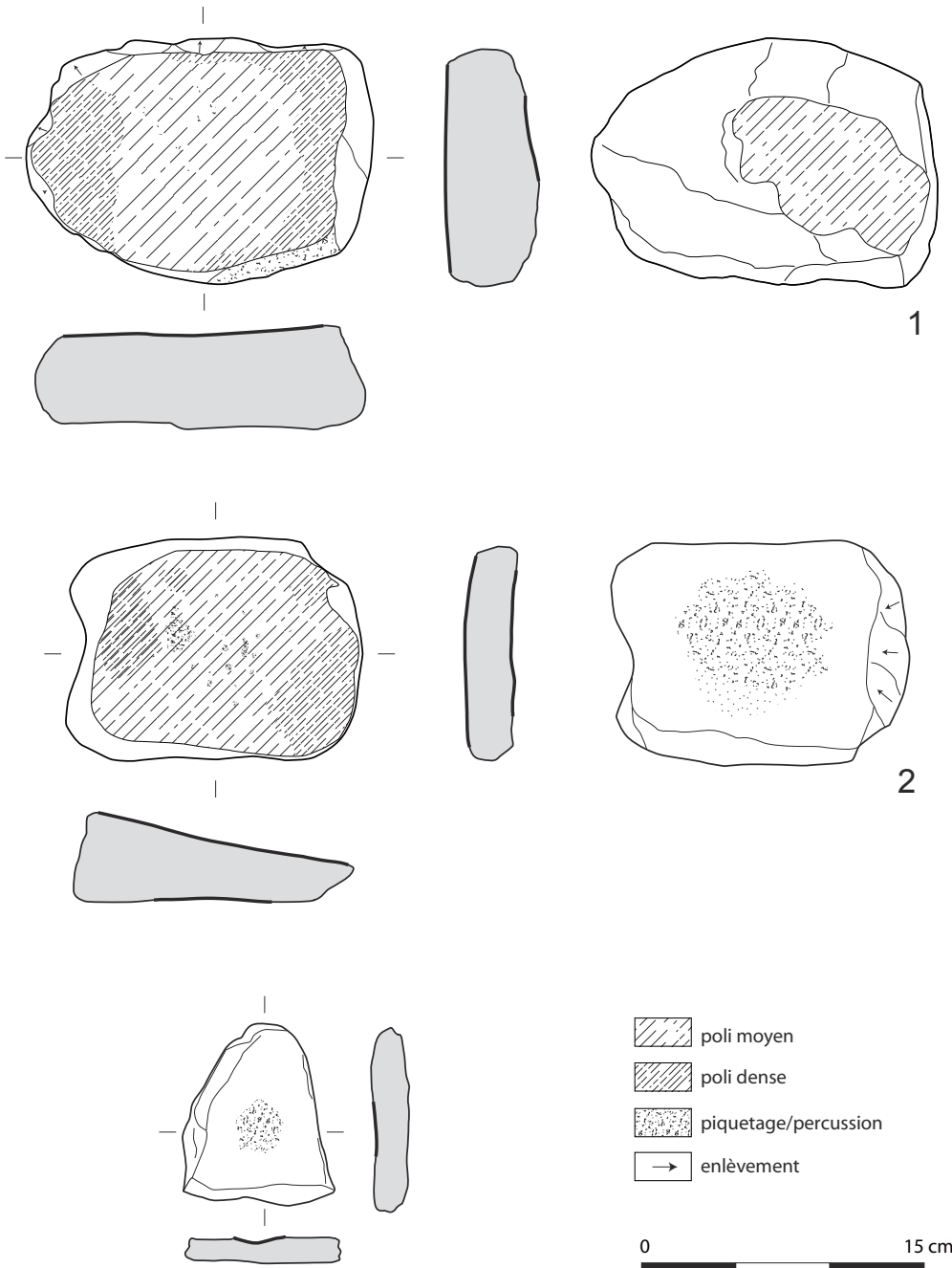

3

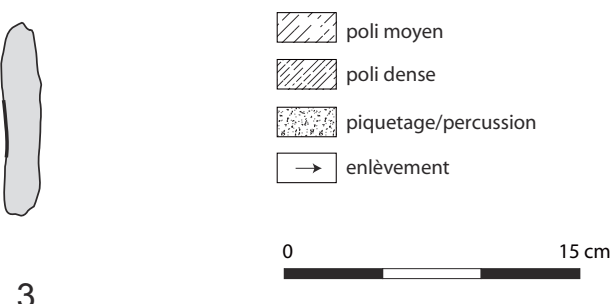

Figure 14 : Vezin-le-Coquet "ZAC des Champs Bleus ». Outillage macrolithique de la structure 7 , Tranchée 168. (C) V. Brisotto, Inrap. Figure 14: Vezin-Le-Coquet, "ZAC des Champs Bleus" (Ille-et-Vilaine). Macrolithic tools from structure 7 , trench 168.

\begin{tabular}{|c|c|c|c|}
\hline Tranchée/Fait/Sondage/US & $N^{\circ}$ Date & Âge BP & Dates calibrées \\
\hline Tr 168 F 2 us 1 & 31542- Lyon -5542 & $4495 \pm 35$ & 3353 à 3028 av. J-C \\
\hline Tr 168 F 2 us 5 & 31543- Lyon- 5553 & $5790 \pm 35$ & 4719 à 4545 av. J-C \\
\hline Tr 168 F 7 sd 3 us 2 & 31540- Lyon -5550 & $5575 \pm 35$ & 4459 à 4346 av. J-C \\
\hline Tr 168 F 9 us 1 & 31541- Lyon - 5551 & $5330 \pm 35$ & 4315 à 4045 av. J-C \\
\hline
\end{tabular}

Tableau 1 : Vezin-le-Coquet «ZAC des Champs Bleus». Datations radiocarbones effectuées au centre de datation par le radiocarbone de Lyon, d'après la courbe M. Stuiver and J. Van der Plicht (éd.), IntCal 2004.

Table 1: Vezin-Le-Coquet, "ZAC des Champs Bleus". Radiocarbon dating by Centre de Datation par le Radiocarbone de Lyon, using the M. Stuiver and J. Van der Plichten (eds) calibration curve, IntCal 2004. 
de Lann Vras au Castellic, d'Er-Lannic à Arzon (Bailloud, 1975), de la Table des Marchand à Locmariaquer (Le Roux, 2006) ou encore du Lizo à Carnac (Bailloud, 1975), et de Saint-Germain à Erdeven (Le Boulaire, Cassen, 2000). Parmi ceux-ci, rares toutefois sont les sites d'habitat comme celui du Lizo.

Par ailleurs plusieurs sites de la côte nord attestent du rayonnement du Castellic en Bretagne. Dans les Côtesd'Armor, le site de Plouedern, Leslouc'h (Blanchet, 2013) a livré plusieurs petits ensembles de fosses sans organisation évidente avec du matériel céramique et lithique comparable à notre assemblage.

Certaines de ces fosses pourraient évoquer un dépôt, comme cela avait déjà été proposé sur le site de Kerléan, à Concarneau pour une fosse isolée qui contenait une coupe à socle, du matériel de mouture et des fragments de noisette carbonisés (Hinguant, 2002; Hamon et Hinguant, 2003). Ces cas ne sont pas rares, puisqu'on retrouve, dans le Morbihan à Kervignac (Henaff et al., 2012) et même au-delà de la Bretagne, comme sur le site de la Burette à Banville, dans le Calvados (Kerdivel, Hamon, 2010), ce même genre de structures isolées avec des assemblages céramiques et lithiques qualifiés de rituels.

À Lamballe, sur le site de la ZAC des Tourelles (Blanchet, 2010) un tronçon de fossé curviligne pourrait se rapprocher de la structure fossoyée F. 168.7 de Vezin-le-Coquet. De même, difficilement repérable au sol, cette structure courbe d'une vingtaine de mètres et d'une largeur moyenne d'un mètre, a livré plusieurs récipients appartenant au Néolithique moyen. Cet assemblage céramique évoque celui des dépôts qui pourraient être d'après les auteurs, d'ordre funéraire ou cérémoniel. Si l'hypothèse d'un enclos ou d'un bâtiment est proposée, plusieurs éléments (espace vide, contournement d'un obstacle aux périodes suivantes) évoquent la présence d'un tertre.

Pour Vezin, aucun indice similaire, ne permet d'envisager la présence d'un tel monument. Par ailleurs, si la nature de l'assemblage céramique peut être comparable, le taux de fragmentation observé ne suggère en aucun cas un dépôt.

Aussi, bien que n'appartenant pas à la sphère du Castellic, le site ceinturé de Lillemer, a livré des formes céramiques attribuées au Néolithique moyen II, comparables à certains éléments de Vezin. Cette occupation semble renvoyer à un statut particulier, avec une configuration et des architectures en briques crues, méconnues jusqu'alors sur la façade atlantique (Laporte et al., 2003).

Au-delà du Massif armoricain, on suppose un rayonnement du Castellic vers le domaine ligérien ainsi qu'en Normandie et dans les îles Anglo-Normandes (Cassen, 2000). Les occupations de Baffrais, Vierville ou Barneville en sont probablement la démonstration (Marcigny et al.,
2010). Pour le domaine méridional, le cas de Sandun à Guérande reste avec Le Lizo à Carnac, un des rares exemples d'habitat pour cette culture (Letterlé et al., 1991; Letterlé, 1997).

L'occupation de Vezin-le-Coquet apparait donc encore à ce jour assez isolée dans le bassin de Rennes quant à cette production céramique.

En ce qui concerne le statut du site et l'organisation des vestiges, encore peu d'exemples nous sont offerts bien que des travaux récents permettent aujourd'hui quelques comparaisons, pour le Néolithique moyen régional.

À Guichen, sur le site de La Croix Vallée (Juhel, 2013) des nébuleuses de trous de poteau mal conservés ont été mis en évidence, livrant une faible quantité de matériel, daté du Néolithique. Les datations radiocarbones ont confirmé et précisé l'hypothèse de bâtiments du Néolithique moyen II.

Pour le site de Kermenguy à Chateauneuf-du-Faou (fouille J.-Y. Tinevez), ce sont plusieurs fosses et trous de poteau qui attestent également d'une architecture contemporaine.

Encore inédits, car de découverte récente, plusieurs bâtiments rectangulaires sur trous de poteau attribués au Néolithique moyen sont en cours d'étude sur les sites de Pléchatel, Le Bois (Juhel, étude en cours), et de la Mézière, Les Lignes de la Gonzée (S. Blanchet, étude en cours).

À Vezin-le-Coquet, si la nature de l'occupation et son organisation spatiale restent difficilement interprétables, la présence d'une tranchée de fondation associée à une série de fosses et de trous de poteau n'est pas sans évoquer les quelques exemples connus d'architectures domestiques se rattachant au Néolithique moyen de l'Ouest (fig. 15). Ainsi, la fouille du dolmen de Cairon a révélé sous le monument funéraire un niveau d'habitat comprenant un bâtiment légèrement trapézoïdal, daté du Néolithique moyen II (Ghesquière et al., 2011).

Ce bâtiment trouve des comparaisons en Angleterre ou encore en Irlande avec les sites de Corbally ou Ballyglass (Smith, 2006).

Plus loin de notre aire géographique, des bâtiments au plan rectangulaire mais au module comparable ont été fouillés à Fossé dans le Cher (Leroy et al., 2007) ou encore à Beaumont Champ-Madame dans le Puy-de-Dôme (Saintot, 2012).

Ainsi, la probable présence d'un bâtiment à Vezin-leCoquet s'ajoute à ces exemples récemment mis en évidence pour le Néolithique moyen régional. Par ailleurs, cette découverte permet de combler une lacune dans l'aire d'extension du Castellic et confirme la présence de réseaux d'échanges, à travers notamment la circulation des matières premières siliceuses. 


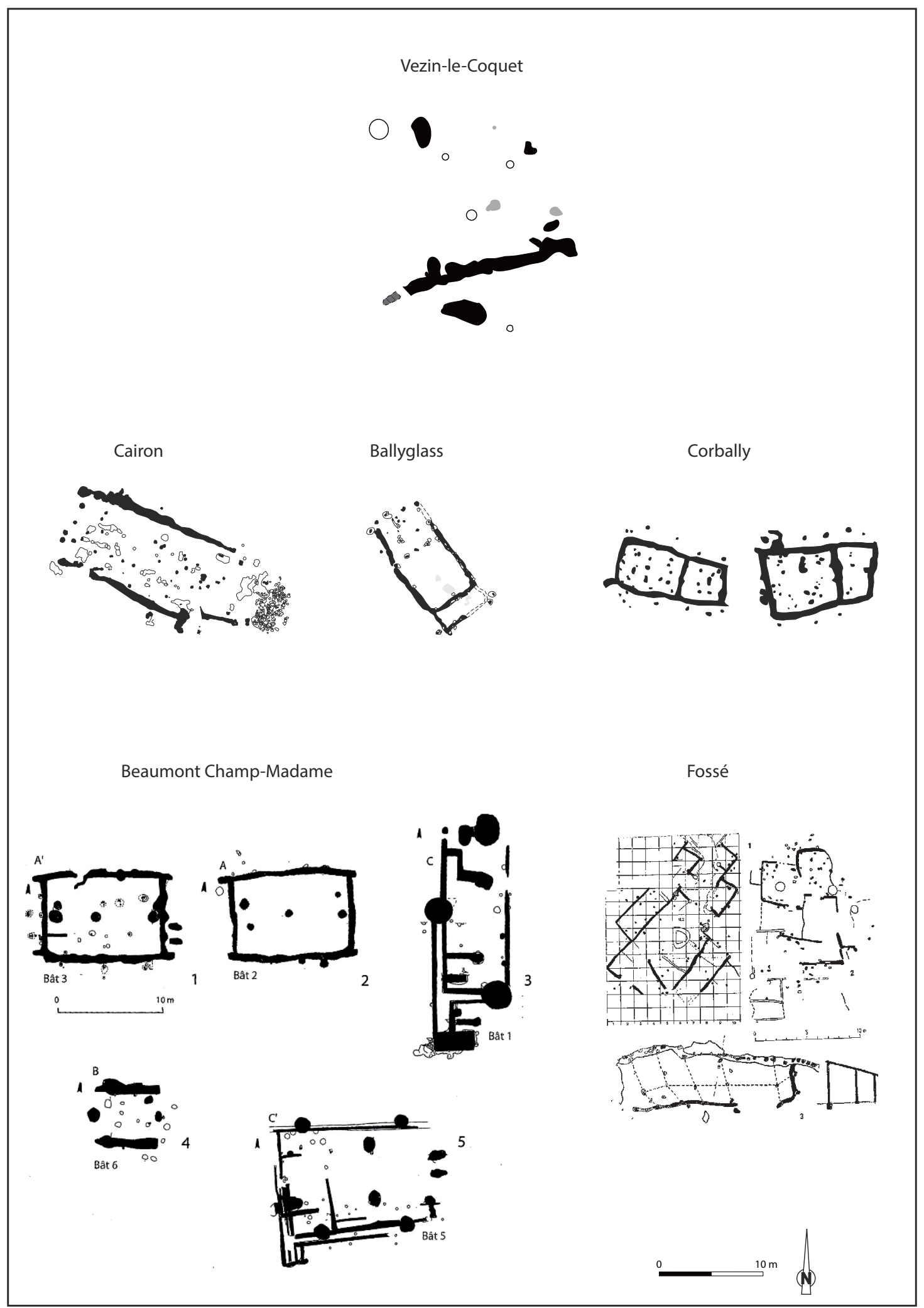

Figure 15: Proposition de comparaisons du plan des vestiges de Vezin-le-Coquet "ZAC des Champs Bleus » avec des plans de bâtiments de la fin du Ve millénaire et du début du IV $V^{e}$ millénaire (d'après Ghesquière et Marcigny, 2011; Smith, 2006; Saintot 2012; Leroy et al., 2007). Figure 15: Comparisons of the site plan of Vezin-le-Coquet "Zac des Champs Bleus" with plans of buildings dating to the end of the $5^{\text {th }}$ Millennium and the beginning of the $\sigma^{\text {th }}$ Millennium. 


\section{Remerciements}

Nous remercions chaleureusement nos collègues Sandie Giovannacci, Sandra Sicard, Grégor Marchand et Jean-Yves Tinevez pour leur précieuse relecture.

\section{Bibliographie}

Aubry L., 20 I I - Pléchatel (Ille-et-Vilaine - Bretagne), Parc d'activités intercommunal, Rapport de diagnostic archéologique, Inrap Grand-Ouest, SRA Bretagne, 59 p.

Bailloud G., 1975 - «Les céramiques “cannelées” du Néolithique armoricain ", Bulletin de la Société Préhistorique Française, t. 72, p. 343-367.

Blanchet S. 2013 - Plouedern (Finistère), Leslouc'h, Une longue occupation de la protohistoire au Moyen Âge, Rapport final d'opération, Inrap Grand ouest, SRA Bretagne, 470 p.

Blanchet S., 20 io - Lamballe, Côtes-d'Armor, ZAC de la Tourelle. Une occupation de longue durée sur le plateau de la Tourelle, Rapport final d'opération, Inrap Grand ouest, SRA Bretagne, $625 \mathrm{p}$.

Blanchet S., 2007 - Betton "Pluvignon" (Ille-et-Vilaine Bretagne) Vol. 1, Le Néolithique ancien, Rapport de fouille préventive, Inrap Grand-Ouest, SRA Bretagne, 290 p.

Brisotto V., 2011 - Vezin-le-Coquet, ZAC "Les Champs Bleus ", tranches $B$ et $C$, Rapport final d'opérations de diagnostic archéologique, Inrap Grand-Ouest, SRA Bretagne, 40 p.

Brisotto V., 2008 - Vezin-le-Coquet, ZAC "Les Champs Bleus ", tranche $D$ (35), Rapport final d'opérations de diagnostic archéologique, Inrap Grand-Ouest, SRA Bretagne, 65 p.

Cassen S., 2011 - «Le Mané Lud en mouvement. Déroulé de signes dans un ouvrage néolithique de pierres dressées à Locmariaquer (Morbihan) ", Préhistoires méditerranéennes, 2, p. 1-58.

Cassen S., 2007 - «Le Mané Lud en images : Interprétations de signes gravés sur les parois de la tombe à couloir néolithique de Locmariaquer (Morbihan) ", Gallia-Préhistoire, 49, p. 197-258

Cassen S., 2000 - "La tradition céramique Castellic ", in Cassen S. et al. (dir.), Éléments d'architecture. Exploration d'un tertre funéraire à Lannec er Gadouer (Erdeven, Morbihan). Constructions et reconstructions dans le Néolithique morbihannais. Propositions pour une lecture symbolique, Chauvigny, Association des publications chauvinoises (mémoire 19), p. 435-459.

Cassen S., Boujot C. et Vaquero Lastres J., 2000 - Éléments d'architecture. Exploration d'un tertre funéraire à Lannec er Gadouer (Erdeven, Morbihan). Constructions et reconstructions dans le Néolithique morbihannais. Propositions pour une lecture symbolique, Chauvigny, Association des publications chauvinoises (mémoire de l'Association des publications chauvinoises; (mémoire 19), p. 29-90.
Cassen S. et Hinguant S., I998 - L'habitat néolithique ancien du Haut-Mée. Saint-Étienne-en-Coglès, Ille-et-Vilaine, autoroute des estuaires, A 84, Rapport de fouille préventive, Afan, SRA Bretagne, $103 \mathrm{p}$.

Cassen S. et L'Helgouac'H J., I 990 - « Du symbole de la crosse : chronologie, répartition et interprétation ", in LE Roux C.-T. (dir.), Paysans et bâtisseurs. L'émergence du Néolithique atlantique et les origines du mégalithisme, Actes du $17^{\mathrm{e}}$ colloque interrégional sur le Néolithique, Vannes, 1990, Rennes : association pour la diffusion des recherches archéologiques dans l'Ouest de la France (Revue archéologique de l'Ouest; suppl. 5), p. 223-235

Dabard M.-P., Marchand G., Monnier J.-L. et Querré G., 2012 - « Roches sédimentaires siliceuses du Massif armoricain, répartition géographique, minéralogie et économie au cours de la préhistoire ", in MARChand G. et Querré G. (dir.), Roches et Sociétés de la préhistoire entre massifs cristallins et bassins sédimentaires, Rennes, PUR, p. 123-137

Escats Y., 20 I - La Mézière, ZAC des lignes de la Gonzée, Rapport final d'opérations de diagnostic archéologique, Inrap GrandOuest, SRA Bretagne.

Ghesquiere E. et Marcigny C., 20 I I - Cairon, Vivre et mourir au Néolithique, La Pierre tourneresse en Calvados, Rennes, PUR, $200 \mathrm{p}$.

Giot P.-R., 1987 - Barnenez, Carn, Guennoc, Rennes, université de Rennes I (Travaux du Laboratoire; 37), 232 p., ill.

Gourio L., 2008 - Étude du mobilier lithique du Néolithique moyen I et II du site de Sandun, commune de Guérande (44), mémoire de master I, université de Rennes 2, Villejean, 125 p.

Grouber P., 2000 - "Les coupes-à-socle d'Er-Lannic (Arzon, Morbihan) ", in Cassen S. (dir.), Eléments d'architecture. Exploration d'un tertre funéraire à Lannec er Gadouer (Erdeven, Morbihan). Constructions et reconstructions dans le Néolithique morbihannais. Propositions pour une lecture symbolique, Chauvigny, Association des publications chauvinoises (mémoire de l'Association des publications chauvinoises; 19), p. 483-527.

Guyodo J.-N., Noslier A., Madioux P. et Bizien-Jaglin C., 200 I - «L'assemblage lithique du site néolithique moyen II de Lillemer (Ille-et-Vilaine) ", Bulletin de la Société préhistorique française 98/4, p. 647-662.

Hamon G., 2003 - «Les productions céramiques au Néolithique ancien et moyen dans le nord-ouest de la France ", Mémoire de thèse, université de Rennes 1, 328 p.

Hamon G. et Hinguant S., 2003 - La coupe à socle de Kerléan (Concarneau, Finistère), Journée civilisations atlantiques et Archéosciences, Rennes, le 9 mars 2002, Rennes, p. 28-30.

Henaff X., Hinguant S., Gaumé E., Colleter R. et Marcoux N., 2012 - "Occupations du Néolithique moyen et de l'âge du Bronze au "Champ du Château" à Kervignac (Morbihan) ", Revue archéologique de l'Ouest, 27, p. 39-72. 
Hinguant S., 2002 - Concarneau (Finistère), Les occupations néolithiques, protohistoriques et médiévales de Kerléan, DFS de fouille archéologique, Inrap Grand-Ouest, SRA Bretagne.

Inuel E., 2004 - «La diffusion du silex du Grand-Pressigny dans le Massif armoricain au Néolithique ", Bulletin des Amis du Musée préhistorique du Grand-Pressigny, Suppl. n 2, 202 p.

IHUEL E., 20 I 2 - «La circulation des lames dans le massif armoricain au Néolithique ", in Marchand G. et Querré G. (dir.), Roches et Sociétés de la préhistoire entre massifs cristallins et bassins sédimentaires, Rennes, PUR, p. 325-340.

Juhel L., 2013 - Guichen, Ille-et-Vilaine, la Croix Vallée, Une occupation du Néolithique moyen et un enclos funéraire du HautEmpire, Rapport final d'opération, Inrap Grand-Ouest, SRA Bretagne, 203 p.

Kerdivel G., Hamon G. et al., 20 io - «Un site du Néolithique moyen, du Néolithique final et de l'âge du Fer à la Burette à Banville (Calvados) : une enceinte du Néolithique moyen II ", in Billard C. et Legris M. (dir.), Premiers Néolithiques de l'Ouest: cultures, réseaux, échanges des premières sociétés néolithiques à leur expansion dans l'Ouest de la France, actes du $28^{\mathrm{e}}$ colloque interrégional sur le Néolithique, Le Havre, 2007, Rennes, PUR, p. 211-235.

Laporte L., Bernard V., Bizien-Jaglin C., Blanchet S., Dietsch-Sellami M.-F., Guitton V., Guyodo J.-N., Hamon G., Madioux P., NaAr S., Nicollin F., Noslier A., Oberlin C. et coll., 2003 - "Aménagements du Néolithique moyen au pied de la butte de Lillemer (Ille-et-Vilaine) : les apports d'un programme de prospection thématique ", Revue archéologique de l'Ouest, 20, p. 127-153.

Le Boulaire A. et Cassen S., 2000 - « Le mobilier néolithique de la collection Chaplain-Duparc (1819-1888). Belz, Erdeven et Plouharnel ", in CAssen S. (dir.), Eléments d'architecture. Exploration d'un tertre funéraire à Lannec er Gadouer (Erdeven, Morbihan). Constructions et reconstructions dans le Néolithique morbihannais. Propositions pour une lecture symbolique, Chauvigny : Association des publications chauvinoises (mémoire de l'Association des publications chauvinoises; 19), p. 461-482.

LE Roux C.-T., 2006 - Gavrinis et les mégalithes du golfe du Morbihan, Paris, Jean-Paul Gisserot éditeur, coll. « Histoire de l'Art ", 112 p.

Le Roux C.-T., Gaumé E., Lecerf Y. et Tinevez J.-Y., 2006 Monuments mégalithiques à Locmariaquer (Morbihan). Le long tumulus d'Er Grah dans son environnement, Paris, CNRS Éditions (Gallia Préhistoire; suppl. 38), 308 p.

Le Rouzic Z., I930 - Carnac. Restaurations faites dans la région. Les cromlechs de Er-Lannic, commune d'Arzon de 1923 à 1926, Vannes, Lafolye \& De Lamarzelle.

Le Rouzic Z., I933 - "Premières fouilles au camp du Lizo (commune de Carnac, Morbihan) », Revue archéologique, p. 189-219.
Leroy D., Ranger O., Verjux C. et Villes A., 2007 - « Réflexion sur l'architecture domestique au Néolihique moyen I dans le Bassin parisien ", in Frère-SAutot M.-C. (éd.), Des trous... Structures en creux pré-et protohistoriques, Actes du colloque de Dijon et Baumes-les-Messieurs, 24-26 mars 2006, p. 187-201.

Letterlé F., Le Gouestre D. et Le Meur N., i99 I - « La chronologie du Néolithique moyen en Armorique à la lumière du site de Sandun à Guérande (Loire-Atlantique) », in BEECHING et al. (dir.), Identité du Chasséen, Actes du Colloque International de Nemours, 1989, Nemours : APRAIF (mémoires du musée de préhistoire d'Île-de-France; 4), p. 149-158.

LetTerlé F., I 997 - «Le Cerny : sa place dans la néolithisation de l'Armorique et le développement des cultures armoricaines au Néolithique moyen I ", in Constantin C., Mordant C. et Simonin D. (dir.), La culture de Cerny. Nouvelle économie, nouvelle société au Néolithique, Actes du colloque international de Nemours, 1994. Nemours, APRAIF (mémoires du musée de préhistoire d'Île-de-France; 6), p. 661-677.

Marcigny C., Ghesquière E., Juhel L. et Charraud F., 2010 - «Entre Néolithique ancien et Néolithique moyen en Normandie et dans les îles Anglo-Normandes ", in BiLlard C. et Legris M. (dir.), Premiers Néolithiques de l'Ouest : cultures, réseaux, échanges des premières sociétés néolithiques à leur expansion dans l'Ouest de la France, Actes du $28^{\mathrm{e}}$ colloque interrégional sur le Néolithique, Le Havre, 2007, Rennes, PUR, p. 117-179.

Mens E., 2006 - «Méthodologie de l'étude technologique des gravures néolithiques armoricaines ", in Joussaume R., LAPORTE L. et Scarre C. (dir.), Origine et développement du mégalithisme de l'Ouest de l'Europe. Actes du colloque international de Bougon, 26-30 octobre 2002, Niort, Conseil général des Deux-Sèvres, p. 721-727.

SAintot S., 2012 - "Continuité et ruptures des ensembles architecturaux et des productions matérielles au cours du Néolithique moyen II à Champ Madame (Beaumont, Puy-deDôme) ", in Perrin T., Sénépart I., Cauliez J., Thirault E. et Bonnardin S. (dir.), Dynamisme et rythmes évolutifs des sociétés de la préhistoire récente, Actualités de la recherche, Actes

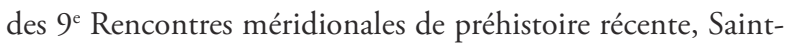
Georges-de-Didonne (17), 8 et 9 octobre 2010, p. 33-49.

SмIтн J., 2006 - The Role of the House in Early Neolithic Ireland, European Journal of Archaeology, vol. 9 (2-3), p. 229-257.

Orton C., Tyers P. et Vince A. G., I 993 - Pottery in Archaeology, Cambridge, Cambridge University Press (Cambridge Manuals in Archaeology), 269 p.

Tournier F., 2006 - Vezin-le-Coquet, ZAC "Les Champs-Bleus", tranche 1, Rapport de diagnostic, Inrap Grand-Ouest, SRA Bretagne, 13 p. 
Zusammenfassung: Spuren einer mittelneolithischen Besiedlung in der Tradition der Castellic-Gruppe im Becken von Rennes (Bretagne, Frankreich) - Die Anlage des Gewerbegebietes „Les Champs bleues“ in der Gemeinde Vezin-le-Coquet (Ille-et-Vilaine, Bretagne, Frankreich) und die in diesem Rahmen zwischen 2006 und 2011 durchgeführten archäologischen Baggerprospektionen haben es gestattet, eine neolithische Besiedlung nachzuweisen. Diese hat, obwohl sie nur schwer eindeutig zu charakterisieren ist, ein keramisches Fundmaterial geliefert, das der Castellic Keramiktradition angehört, sowie ferner ein kleines lithisches und makrolithisches Steingeräteinventar, das einer regionalen Ausprägung des Mittelneolithikums-II zugewiesen werden kann. Diese Steingeräteserie ist bislang im Becken von Rennes einzigartig. Sie gestattet es, die geographische Verbreitung dieser Kulturstufe in einem Gebiet näher einzugrenzen, das auf halbem Wege zwischen den Fundplätzen des Ausgangsgebietes um den Golf von Morbihan herum und denjenigen der Nordküste liegt. Ferner gibt es einige archäologische Hinweise auf ihre Verbreitung in der Normandie sowie auf den britischen Kanalinseln.

Resumen: Las huellas de una ocupación del Neolitico medio de tradición Castellic en la cuenca de Rennes (Bretaña, Francia) - Consiguiente a la creación de las ZAC des Champs-Bleus, en la ciudad de Vezin-le-Coquet (Ille-et-Vilaine), las diversas evaluaciones arqueológicas llevadas a cabo entre 2006 y 2011 ayudaron a identificar una ocupación neolitica que, aunque sea difícil de caracterizar, ha entregado restos de cerámica de tradición Castellic y un pequeño conjunto litico y macro-lítico que puede relacionarse con el Neolítico medio II regional. Esta serie es la primera que se encuentra en la cuenca de Rennes. Permite colocar un hito geográfico de este periodo, a medio camino entre los sitios de la zona nuclear localizada alrededor del Golfo de Morbihan, los de la costa norte, y los escasos indices que acreditan de su influencia en Normandia y las Islas del Canal.

Schlüsselwörter: Mittelneolithikum, Bretagne, Castellic, Keramik, Steingeräte, Makrolitisches Steingeräteinventar, Gebäude.

Palabras clave: Neolítico medio, Bretaña, Castellic, cerámica, herramientas de piedra y macro-lítico, edificio. 